\title{
PSYCHE Pure Shift NMR Spectroscopy
}

DOI:

10.1002/chem.201800524

\section{Document Version}

Accepted author manuscript

Link to publication record in Manchester Research Explorer

\section{Citation for published version (APA):}

Foroozandeh, M., Morris, G., \& Nilsson, M. (2018). PSYCHE Pure Shift NMR Spectroscopy. Chemistry: A European Journal . https://doi.org/10.1002/chem.201800524

\section{Published in:}

Chemistry: A European Journal

\section{Citing this paper}

Please note that where the full-text provided on Manchester Research Explorer is the Author Accepted Manuscript or Proof version this may differ from the final Published version. If citing, it is advised that you check and use the publisher's definitive version.

\section{General rights}

Copyright and moral rights for the publications made accessible in the Research Explorer are retained by the authors and/or other copyright owners and it is a condition of accessing publications that users recognise and abide by the legal requirements associated with these rights.

\section{Takedown policy}

If you believe that this document breaches copyright please refer to the University of Manchester's Takedown Procedures [http://man.ac.uk/04Y6Bo] or contact uml.scholarlycommunications@manchester.ac.uk providing relevant details, so we can investigate your claim.

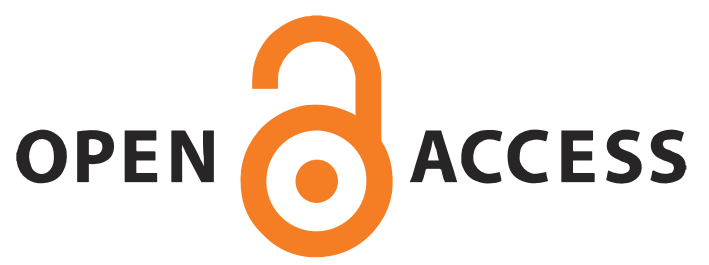




\section{CHEMISTRY A European Journal}

\section{Accepted Article}

Title: PSYCHE Pure Shift NMR Spectroscopy

Authors: Mohammadali Foroozandeh, Gareth Morris, and Mathias Nilsson

This manuscript has been accepted after peer review and appears as an Accepted Article online prior to editing, proofing, and formal publication of the final Version of Record (VoR). This work is currently citable by using the Digital Object Identifier (DOI) given below. The VoR will be published online in Early View as soon as possible and may be different to this Accepted Article as a result of editing. Readers should obtain the VoR from the journal website shown below when it is published to ensure accuracy of information. The authors are responsible for the content of this Accepted Article.

To be cited as: Chem. Eur. J. 10.1002/chem.201800524

Link to VoR: http://dx.doi.org/10.1002/chem.201800524 


\section{PSYCHE Pure Shift NMR Spectroscopy}

Mohammadali Foroozandeh, Gareth A. Morris, and Mathias Nilsson*

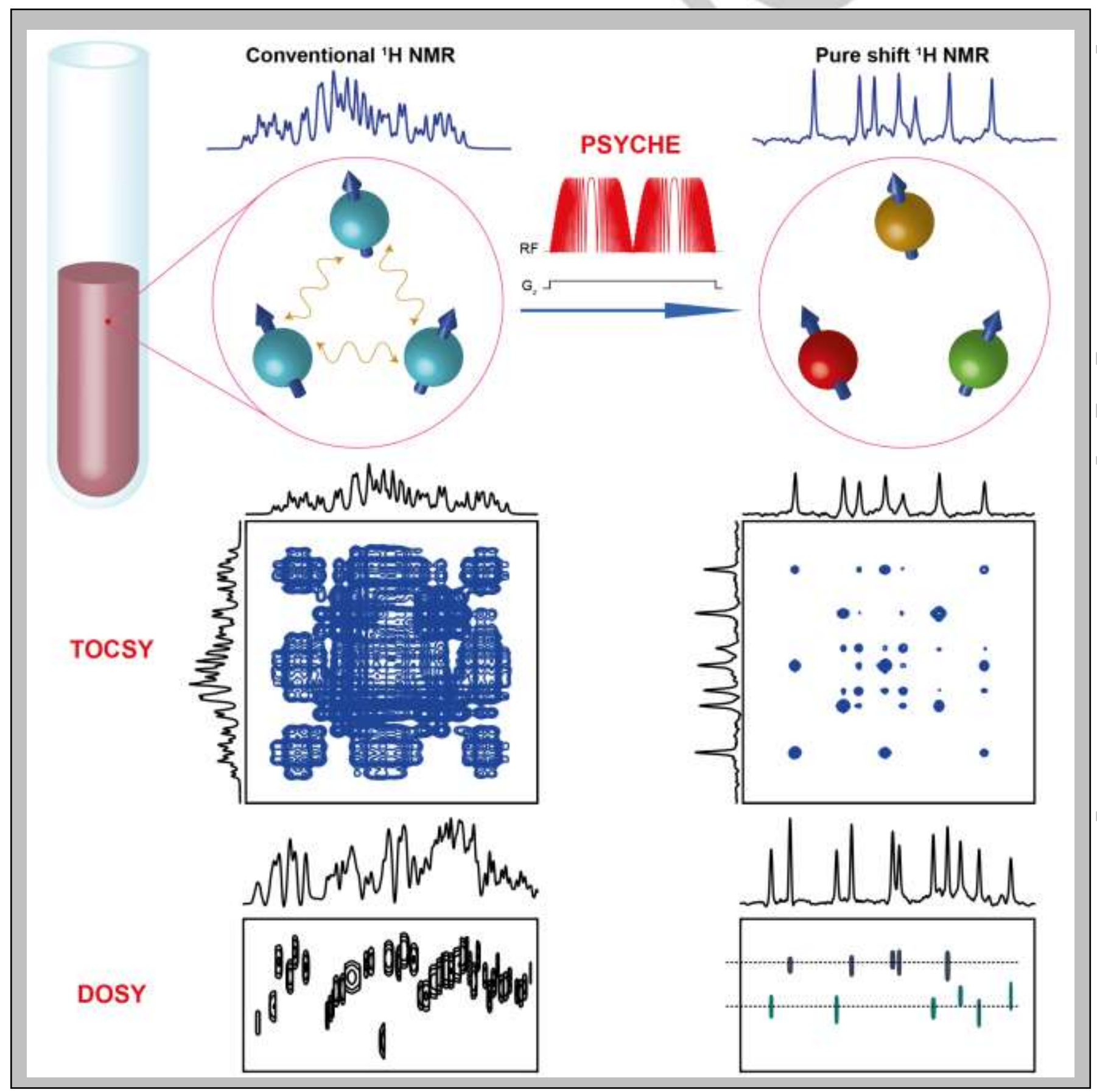


Abstract: Broadband homodecoupling techniques in NMR, also known as "pure shift" methods, aim to enhance spectral resolution by suppressing the effects of homonuclear coupling interactions to turn multiplet signals into singlets. Such techniques typically work by selecting a subset of "active" nuclear spins to observe, and selectively inverting the remaining, "passive", spins to reverse the effects of coupling. Pure Shift Yielded by Chirp Excitation (PSYCHE) is one such method; it is relatively recent, but has already been successfully implemented in a range of different NMR experiments. Paradoxically, PSYCHE is one of the trickiest of pure shift NMR techniques to understand but one of the easiest to use. Here we offer some insights into theoretical and practical aspects of the method, and into the effects and importance of the experimental parameters. Some recent improvements that enhance the spectral purity of PSYCHE spectra will be presented, and some experimental frameworks including examples in 1D and 2D NMR spectroscopy, for the implementation of PSYCHE will be introduced.

\section{Introduction and background}

Resolution and sensitivity are key to accessing information from NMR spectra, and have been the focus for improvement since the dawn of NMR spectroscopy. We have certainly become much better off with regard to sensitivity in recent years, largely due to the introduction of cryogenic probes, but spectral resolution lags behind. It can still be a difficult task to interpret NMR spectra of complex systems, and there is an urgent need for better resolution. In the case of ${ }^{1} \mathrm{H}$ NMR, the most common NMR experiments in both academia and industry, the combination of the narrow range of the proton chemical shift (ca. $10 \mathrm{ppm}$ ) with the extensive multiplet structure due to scalar coupling frequently result in signal overlap, which makes spectral analysis and assignment complicated and sometimes impossible. Multiplet structure can arise from both homonuclear (e.g. $\left.{ }^{1} \mathrm{H}-{ }^{1} \mathrm{H}\right)$ and heteronuclear (e.g. ${ }^{19} \mathrm{~F}-{ }^{1} \mathrm{H}$ ) couplings. The latter can conveniently be suppressed, if required, by applying continuous irradiation, selective or broadband, of the heteronuclei during signal acquisition or evolution. For homonuclear couplings, however, the story is completely different and subtler manipulations of the spins are required. Over the last two decades, the belated advent of broadband homonuclear decoupling methods, long sought and long feared to be unattainable, has finally led to a series of developments in so-called "pure shift" NMR methods ${ }^{[1]}$. These methods yield spectra containing only a single signal for each chemically distinct site, leading to very significant improvements in spectral resolution, by suppressing the effects of homonuclear couplings. One unfortunate drawback of most of these methods is a substantial cost in sensitivity, typically of one to two orders of magnitude.

Dr M. Foroozandeh, Prof G. A. Morris, and Dr M. Nilsson School of Chemistry, University of Manchester, Oxford Road, Manchester M13 9PL, UK.

E-mail: mathias.nilsson@manchester.ac.uk
Broadband homodecoupling methods rely on distinguishing between the interactions of spins with the main magnetic field (chemical shifts) and interactions with each other (couplings). Different methods use different approaches to achieve this. The earliest approach in liquid state NMR was to use the spin echo in a two-dimensional experiment (so-called 2D J, or J-resolved, spectroscopy ${ }^{[2]}$ ). Here the spin echo suppresses the effect of the chemical shift while leaving that of homonuclear couplings unaffected, allowing the effect of the couplings on the free induction decays $S\left(t_{2}\right)$ to be mapped out as a function of the incremented evolution time $t_{1}$. Double Fourier transformation as a function of $t_{1}$ and $t_{2}$ then yields a spectrum in which signals are dispersed in $F_{1}$ as a function of couplings only and in $F_{2}$ as a function of both couplings and chemical shifts. A $45^{\circ}$ projection of the $2 \mathrm{D}$ spectrum, along the direction in which the multiplet structure is spread out, then gives access to the chemical shiftonly spectrum. Unfortunately, in the classic 2DJ experiment this projection has to be carried out in absolute value mode because the data are phase modulated in both dimensions. If the data are projected in phase sensitive mode, positive and negative signal contributions cancel to give a zero projection. The use of absolute value processing introduces gross nonlinearities, and ends up sacrificing most of the anticipated resolution advantage.

Despite the disadvantages of the 2DJ approach, it prompted a search for other pulse sequence elements that distinguish between shifts and couplings, and in particular for elements that do the opposite of the spin echo, refocusing the effects of scalar couplings while allowing those of chemical shifts to continue to evolve. Typically, such "J-refocusing" pulse sequence elements work by selecting a small subset of spins (the active spins) from the ensemble and suppressing the effect on those spins of any couplings with the remaining (passive) spins. Placed at the middle of an evolution period $t_{1}$, a J-refocusing sequence element ideally allows the chemical shift evolution to be mapped out independent of the effects of homonuclear scalar couplings. A number of different principles have been exploited to achieve J-refocusing: the Zangger-Sterk (ZS) method ${ }^{[3]}$ selects the active spins using a selective $180^{\circ}$ pulse in the presence of a weak field gradient; the BIRD method ${ }^{[4]}$ selects those spins directly bonded to a lowabundance heteronucleus such as ${ }^{13} \mathrm{C}$; and the PSYCHE method $^{[5]}$, the subject of this article, uses low flip angle frequencyswept ("chirp") pulses in the presence of a field gradient both to select a small proportion of the spins and to suppress responses from the remaining, passive, spins.

The ZS decoupling scheme has found application in many oneand two-dimensional NMR experiments including $1 \mathrm{D}^{1} \mathrm{H} \mathrm{NMR}^{[6]}$, DOSY, ${ }^{[7]}$ TOCSY[6c, 8], NOESY[9], and 2D J spectroscopy ${ }^{[10]}$. Because the combination of the selective $180^{\circ}$ pulse and field gradient results in only a thin slice of sample being excited for any given chemical shift, it incurs a large but variable sensitivity penalty that is proportional to the ratio of the bandwidth of the frequency-selective pulse to the spectral window of interest. The method only works where the difference in chemical shift between coupled spins is greater than the bandwidth of the selective pulse used, so the sensitivity penalty is greatest where coupled spins are close in chemical shift. Band-selective (BS) homodecoupling methods ${ }^{[11]}$ can be seen as a special case of the ZS method in 
which no field gradient is applied during the frequency-selective $180^{\circ}$ pulse. This means that the active spins are chosen solely on the basis of chemical shift: only couplings between active and passive spins are suppressed, any couplings between spins that both experience the $180^{\circ}$ selective pulse survive. Although this approach does not result in a broadband homodecoupled spectrum, typically only exciting a small proportion of the chemical shift range, it does give access to homodecoupling at full sensitivity since no slice selection is used.

The BIRD method has found some application in $1 \mathrm{D} 1 \mathrm{H} \mathrm{NMR}{ }^{[4}$ ${ }^{12]}$, particularly for spin systems that are strongly coupled in ${ }^{12} \mathrm{C}$ isotopomers, but as it mostly uses ${ }^{13} \mathrm{C}$ at natural abundance it incures a constant sensitivity penalty of just over two orders of magnitude. However, in experiments such as HSQC that only observe ${ }^{13} \mathrm{C}$-isotopomers this sensitivity penalty has been paid in advance, and the use of the BIRD J-refocusing element becomes very attractive. Indeed if the BIRD element is used in real-time pure shift acquisition mode, in which $\mathrm{J}$-refocusing elements are applied repeatedly during acquisition of free induction decays, pure shift HSQC gives a net sensitivity advantage over conventional ${ }^{1} \mathrm{H}^{13} \mathrm{C}$ HSQC at natural abundance ${ }^{[13]}$ or ${ }^{1} \mathrm{H}-{ }^{15} \mathrm{~N}$ HSQC in proteins ${ }^{[14]}$.

Experimental pure shift data are more usually acquired using a series of experiments in which free induction decays $S\left(t_{2}\right)$ are acquired as a function of a pure shift evolution time $t_{1}$, and a pure shift "interferogram" is constructed by concatenating short chunks of data points taken from successive free induction decays. Whether the chunks are acquired discretely, or continuously in real time ${ }^{[6 \mathrm{c}, 11 \mathrm{a}, 11 \mathrm{c}]}$, since the evolution of scalar coupling is much slower than that of the chemical shift, during a short chunk[6a] of data acquisition the evolution of scalar couplings can be largely neglected, so when a series of such chunks are stitched together the result is a pure shift FID.

A third type of implementation of pure shift NMR is in the indirect dimension of $2 \mathrm{D}$ experiments ${ }^{[15]}$. Heteronuclear shift correlation spectra without (homonuclear) multiplet structure have been available for many years. For example, in the classic ${ }^{13} \mathrm{C}$ observed HETCOR experiment, BIRD can be used to suppress ${ }^{1} \mathrm{H}-{ }^{1} \mathrm{H}$ couplings in the $F_{1}$ dimension ${ }^{[16]}$. An even older approach is the constant-time method ${ }^{[15]}$, in which the evolution of the chemical shift is mapped out by moving a $180^{\circ}$ pulse incrementally within a constant delay. A more recent example of broadband homonuclear decoupling in an indirect dimension is the use of the ZS method to decouple the carbon domain of an HSQC experiment for per- ${ }^{13} \mathrm{C}$ samples, since here the BIRD method is not applicable due to the full ${ }^{13} \mathrm{C}$ enrichment [17].

While PSYCHE has been widely adopted, and is simple to implement and use, it is perhaps less widely understood in detail. The aim of the present review is to give the reader an insight into the most relevant theoretical and practical aspects of the PSYCHE method, to shed light on some of the subtleties involved and their practical implications, and to illustrate some of the ways in which it can be applied. Some recent improvements, and also some experimental frameworks which can benefit from this method in future implementations, will also be discussed.

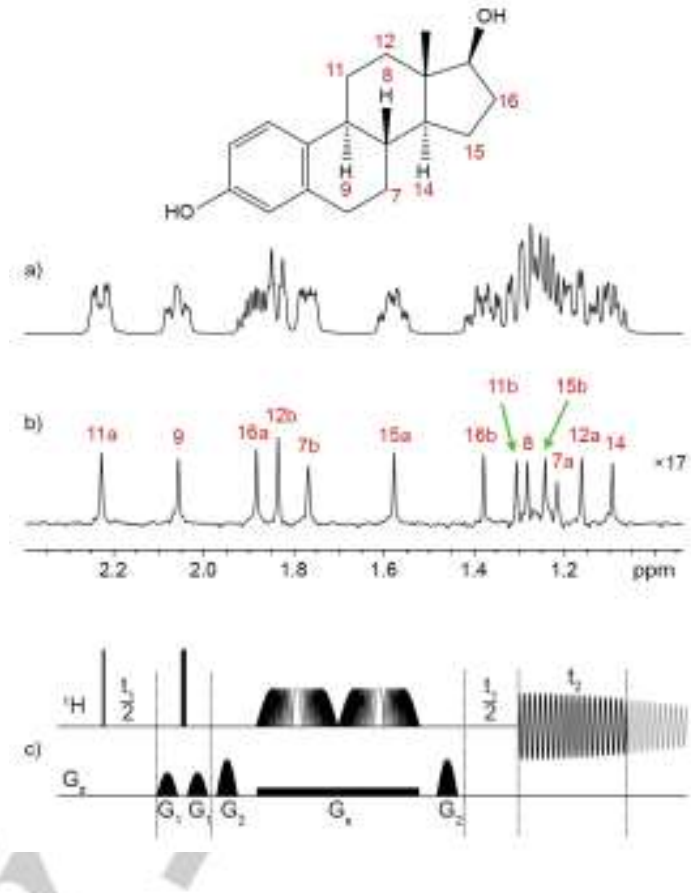

Figure 1. Proton spectra of a sample of estradiol (scheme) in DMSO- $d_{6}$, acquired by (a) conventional ${ }^{1} \mathrm{H}$ NMR and (b) PSYCHE. c) Pulse sequence for 1D PSYCHE pure shift NMR; narrow rectangles are $90^{\circ}$ r.f. pulses, wide are $180^{\circ}$ pulses, and trapezoids are low-power saltire chirp pulses of nominal flip angle $\beta<<90^{\circ}$.

\section{PSYCHE decoupling}

Most of the current techniques used for measuring pure shift spectra, including constant-time, BIRD, ZS and BS experiments, are constructed from relatively familiar components and have been well described in recent reviews on pure shift $\mathrm{NMR}^{[1]}$ Homodecoupling methods such that use low flip angle pulses, however, such as anti $z-\mathrm{COSY}^{[18]}$ and PSYCHE, have until recently attracted much less attention. In this section, a simple account of the mechanics of the PSYCHE method will therefore be given.

The PSYCHE pulse sequence element (Figure 2c) consists of two low flip angle $(\beta)$ swept-frequency pulses, in the presence of a weak pulsed field gradient. Although the experiments look very different, there is a close analogy with the basic anti z-COSY experiment of Pell, Edden and Keeler, ${ }^{[18]}$ which can be treated as consisting of a small flip angle stimulated echo and a hard $180^{\circ}$ pulse. In both cases the overall effect of the low flip angle pulses is to refocus the effects of $\mathrm{J}$-couplings on a small proportion $\sin ^{2} \beta$ of the spins in the sample, the active spins. The principal difference between the two methods lies in how the unwanted signals from the remaining proportion $\cos ^{2} \beta$, the passive spins, are rejected. The interfering signals include COSY-type, ${ }^{[18 \mathrm{~b}]}$ zero quantum, ${ }^{[19]}$ and strong coupling responses ${ }^{[20]}$. In anti z-COSY they are treated by acquiring an excess of data and then removing the unwanted responses by post-processing; in PSYCHE, frequency-swept pulses in the presence of weak field gradients are used to suppress them directly by spatiotemporal averaging. 
Let us start with some simplifications and useful approximations. Although the effects of the PSYCHE sequence element in space and time are interdependent, for the sake of simplicity we divide the effect of the element into two separate modes of action, temporal and spatial. For ease of illustration we assume that the effect of a swept-frequency (chirp) pulse can be approximated by an instantaneous spin flip through an angle $\beta$ at a time that depends on the Larmor frequency. Thus different spins with different offsets are flipped instantaneously at different times. In PSYCHE, we have a pair of chirp pulses in which the frequencies sweep linearly with time in opposite directions. If the chirp pulses sweep through a range of frequencies $\Delta F,(\Delta F / 2$ either side of the transmitter frequency) in a time $\tau_{p}$, then a spin $A$ with an offset $\Delta v_{A}$ from the transmitter will experience two rotations $\beta$, at times $(0.5+\alpha) \tau_{p}$ and $(0.5-\alpha) \tau_{p}$ where $\alpha=\left(\Delta v_{A} / \Delta F\right)$. Thus spins with different offsets from resonance experience low flip angle stimulated echoes with different timings.

We can decompose the mechanism of PSYCHE into three parts, which will be discussed below: 1) the effect of the flip angle $\beta$ on multiplet patterns, and the relationship between PSYCHE and anti z-COSY; 2) the temporal effect of the swept-frequency (chirp) pulses; and finally 3 ) the spatial effect of the weak pulsed field gradient during the PSYCHE pulse.

a)
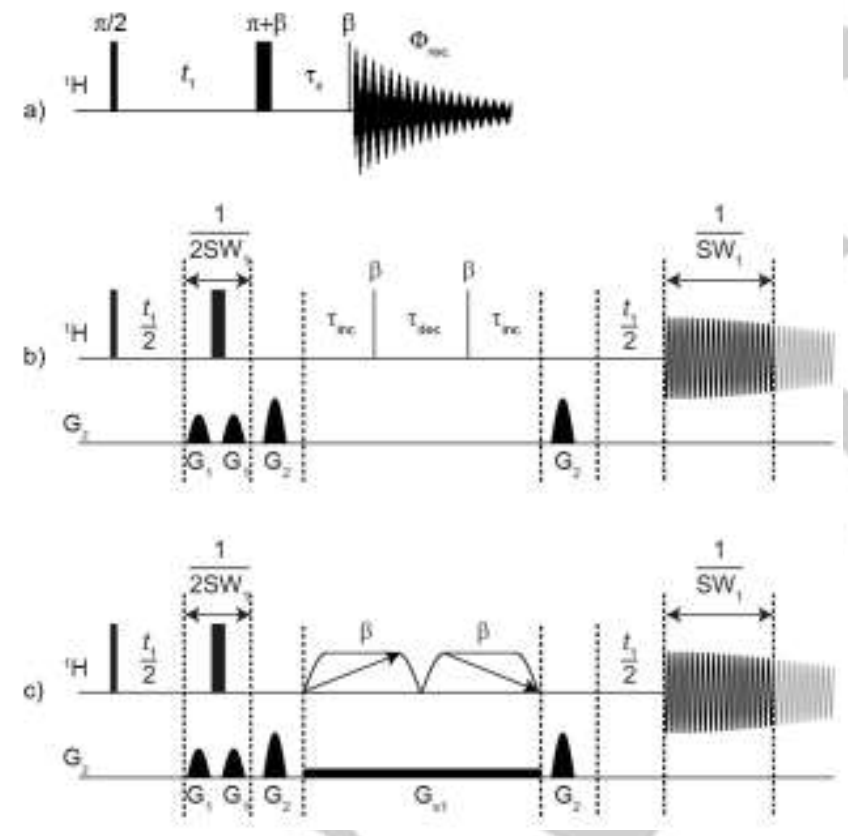

Figure 2. Pulse sequence diagrams of (a) anti z-COSY, (b) simplified model of PSYCHE based on anti Z-COSY, and (c) PSYCHE. Delays $T_{\text {inc }}$ and $T_{\text {dec }}$ indicate incremented and decremented delays respectively to demonstrate the effect of two counter-sweeping chirp pulses taking advantage of instantaneous flip approximation.

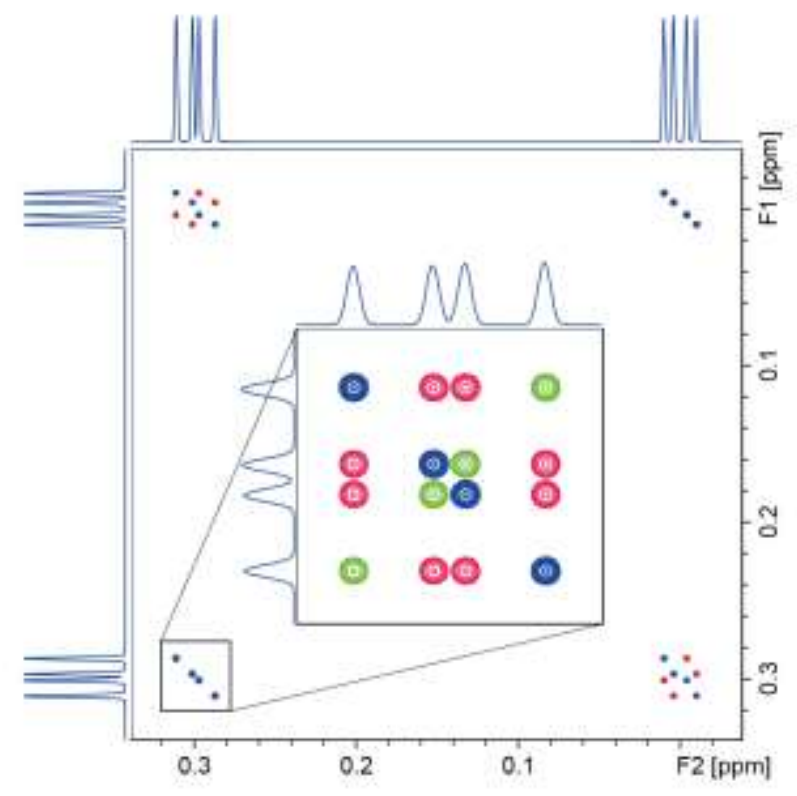

Figure 3. Part of the anti Z-COSY spectrum of an AMX spin system. Simplification of cross-peaks and diagonal multiplets results from the use of lowflip angle pulses. Insert shows the color-coded classes of diagonal peak multiplet components which have different flip angle dependences, as shown in Figure 4.

\section{Low flip angle pulses and homodecoupling}

The anti $z$-COSY pulse sequence (Figure 2a) has a similar structure to the conventional two-pulse COSY pulse sequence, with the difference that the mixing period contains the sequence $\left(\beta+180^{\circ}\right)-T_{z}-\beta$ instead of a single pulse. In the original anti $z-$ COSY experiment, low-flip angle pulses were used to simplify the COSY spectrum and facilitate identification of coupling partners and $\mathrm{J}$-coupling values and signs ${ }^{[18 a]}$. Because of the $180^{\circ}$ pulse, in the limit of small flip angle $\beta$ only multiplet components which correspond to opposite passive spin states in $t_{1}$ and $t_{2}$ survive in the cross- and diagonal-peak multiplets in the resultant spectrum. If $\beta$ is small enough, the diagonal peaks consist simply of the 1D multiplet spread out along a line perpendicular to the main diagonal (Figure 4f). Pell, Edden and Keeler used this beautiful property to get access to a pure shift spectrum, by using direct projection of the $2 \mathrm{D}$ diagonal peaks of a phase-sensitive anti $z$ COSY spectrum at right angles to the diagonal[18b].

The dependence of the multiplet structure of a diagonal peak on the flip angle $\beta$ in anti $z$-COSY is shown in Figure $\mathbf{3}$ and Figure 4. Here we are looking at one diagonal peak from the anti z-COSY spectrum (Figure 3 ) of a three-spin system. We can identify three different categories of signal: i) diagonal (green), ii) anti-diagonal (blue), and iii) off-diagonal (red) (insert in Figure 3). As can be seen, reducing the flip angle $\beta$ results in further and further simplification of the 2D multiplet, leaving us with just the signals that lie along the anti-diagonal of the 2D multiplet. However, this simplicity comes at the price of greatly reduced signal amplitude, so a compromise is needed between sensitivity and spectral 
purity. If $\beta$ is too high, the presence of signals other than those lying along the anti-diagonal will lead to extra peaks, known as recoupling artefacts, appearing either side of the pure shift signals in the projected spectrum. A flip angle $\beta$ of $10^{\circ}$ to $30^{\circ}$ is generally suitable for the majority of samples.

In the basic anti z-COSY experiment a pure shift spectrum is obtained by $2 \mathrm{D}$ processing as normal, then removing all peaks outside the diagonal region and projecting onto the diagonal (i.e. integrating the spectrum along an axis at right angles to the diagonal). In PSYCHE, on the other hand, the double chirp PSYCHE pulse sequence element and a hard $180^{\circ}$ pulse are placed at the midpoint of an evolution time $t_{1}$ and a pure shift free induction decay is constructed from chunks of data of duration $1 / s w_{1}$ acquired for successive increments of $1 / s w_{1}$ in $t_{1}$. The coherence transfer pathways that would have led to off-diagonal peaks in the anti z-COSY experiment are suppressed by spatiotemporal averaging in PSYCHE.
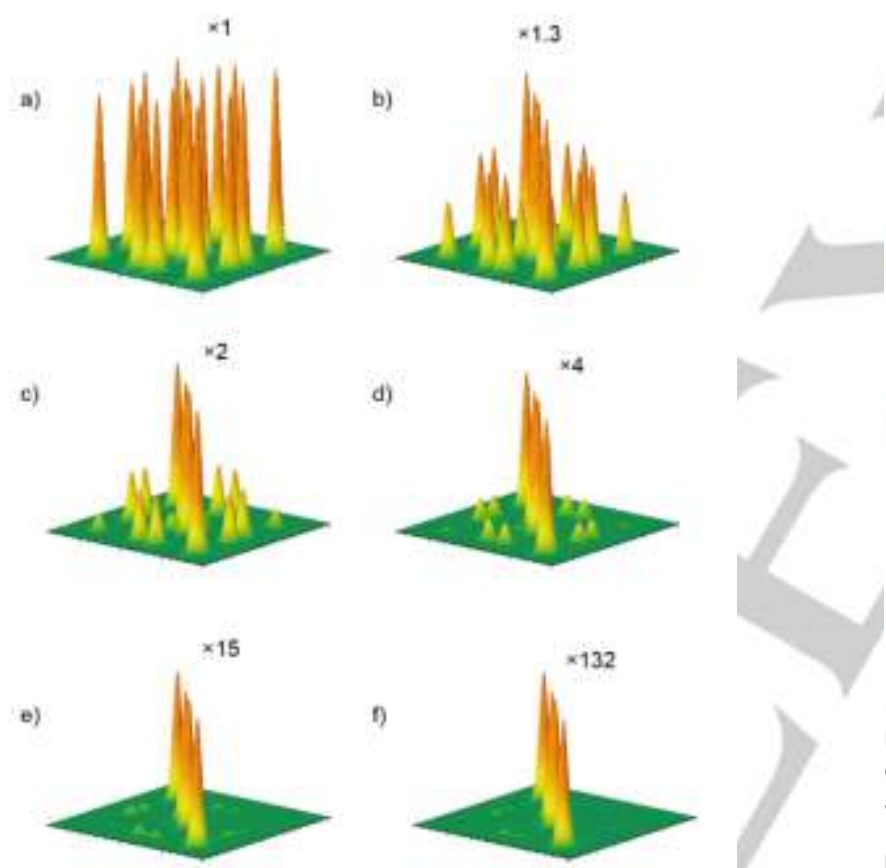

that the relationship between the effective flip angle of a chirp pulse and its radiofrequency amplitude is not as simple as that for rectangular hard pulses, where the flip angle is directly proportional to the amplitude and duration of a pulse.

Let us start with the simple case of a unidirectional chirp pulse, sweeping linearly through a range of frequencies $\Delta F$ over a duration $\tau_{p}$. The relationship between the radiofrequency amplitude r.f. in $\mathrm{Hz}$ and the flip angle $(\beta)$ of such a pulse can be written:

$$
r . f .=\sqrt{\frac{\tau_{p} \times \Delta F \times Q}{2 \pi}} / \tau_{p}, \text { where } Q=-2 \times \ln \left(\frac{\cos (\beta)+1}{2}\right) / \pi
$$

The flip angle approaches $180^{\circ}$ asymptotically - the adiabatic limit - as the radiofrequency amplitude increases so in principle a perfect $180^{\circ}$ rotation is not achievable. In practice, a $Q$-factor of 5 is sufficient to give good inversion and refocusing performance. For smaller flip angles, the radiofrequency amplitude required can be calculated from the required bandwidth, duration, and flip angle using the equation above. a)

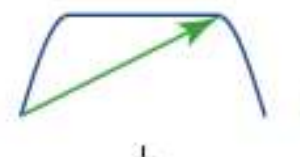

$+$

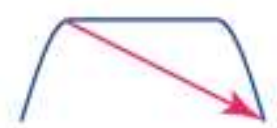

$\downarrow$

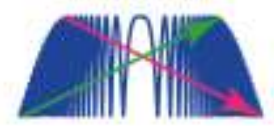

b)
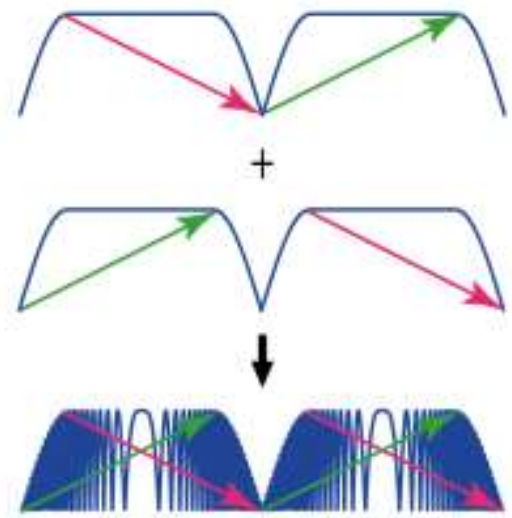

Figure 5. Representations of unidirectional and saltire chirp pulses. (a) Shows how two counter-sweeping unidirectional chirp pulses can be combined to generate a saltire pulse; blue lines represent the amplitude profiles of the pulses and coloured arrows represent the direction of the frequency sweep: low-to-high (green) and high-to-low (red). (b) Shows the PSYCHE pulse element which can be generated simply by concatenation of two saltire chirp pulses. How this pulse acts as two individual pulses at the same time is shown in Figure 7.

Figure 4. Effect of varying the flip angle $\beta$ on the multiplet structure of a diagonal signal in the anti z-COSY spectrum of an AMX spin system. The flip angles of the two $\beta$ pulses in PSYCHE element were set to (a) $90^{\circ}$, (b) $60^{\circ}$, (c) $45^{\circ}$, (d) $30^{\circ}$, (e) $15^{\circ}$, and (f) $5^{\circ}$. The number above each spectrum indicates the relative vertical scaling and hence indicates the amount of sensitivity lost due to the use of low-flip angle pulses.

\section{Swept-frequency pulses and temporal effects}

Swept-frequency 'chirp' pulses have been known and applied in NMR for a long time, but until recently their applications have mainly been limited to broadband inversion and refocusing. The PSYCHE method, in contrast, uses such pulse shapes for low flip angle excitation. One potential source of confusion here is the fact
Although PSYCHE works well with conventional, unidirectional, chirp pulses, significantly improved performance in PSYCHE can be obtained by using saltire chirp pulses. A saltire pulse is the average of two counter-sweeping unidirectional chirp pulses (Figure 5), i.e. it is a pulse which simultaneously sweeps in frequency linearly in opposite directions over a frequency range $\Delta F$ in a time $\tau_{p}$. As shown in Figure 5, this averaging changes the pulse from phase-modulated to amplitude modulated, modifying the appearance of the amplitude envelope. This also simplifies the relationship between flip angle, radiofrequency amplitude and duration. For resonances well within the range $\Delta F$ 
(so that the effect of smoothing of the pulse envelope to zero at either end can be neglected) the relationship is:

$$
r . f .=(\beta / 360) \sqrt{2 \times \Delta F / \tau_{p}}
$$

which shows a linear relationship between flip angle, radiofrequency amplitude and sweep rate $\left(\Delta F / \tau_{p}\right)$. As we will see in the next section, the sweep rate is important in achieving efficient suppression of unwanted signals.

While the linear relationship between radiofrequency amplitude and flip angle is convenient, the principal advantage of using saltire pulses is that they improve the discrimination between antidiagonal and off-diagonal peaks in Figures 2 and 3 , and hence between pure shift and recoupling signals in the resultant spectrum. As shown in Figure 7, the evolution of coherences during a saltire PSYCHE element can be represented as the superposition of two independent coherence transfer pathways (CTPs), each enforced by the presence of the encoding gradient during the pulses. Thus the effect of using the saltire pulse element is equivalent to the simultaneous application of two PSYCHE elements in which the sweep directions of the pulses are reversed. The advantage of using saltire pulses instead of unidirectional chirps can be simply illustrated with an AX spin system. The principal type of artefact encountered in a PSYCHE experiment arises from coherence transfer in which both coupled spins, rather than just one, are excited by the $\beta$ pulses. For unidirectional chirp pulses, the amplitudes of these artefactual signals are proportional to $\sin ^{4} \beta$, while the pure shift signals have amplitudes proportional to $2 \sin ^{2} \beta \cos ^{2} \beta$. Using saltire chirp pulses changes these expressions to $2 \sin ^{4}(\beta / 2)$ and $4 \sin ^{2}(\beta / 2) \cos ^{2}(\beta / 2)$ respectively, so that the spectral purity (the ratio of wanted to unwanted signal intensity) increases by a factor of four. (The different types of coherence transfer pathway excited in PSYCHE experiments are discussed in more detail below). Automatic calibration of saltire pulse shapes based on userdefined parameters can easily be incorporated into practical pulse sequences, a number of which are available for free download from http://nmr.chemistry.manchester.ac.uk/?q=node/255.

\section{Field gradients and spatial effects}

In general in pure shift spectra, and in particular in the case of PSYCHE experiments, observed signals can be categorised into three types: 1) pure shift signals, which carry only chemical shift information; 2) intrinsic artefacts, e.g. recoupling signals caused by of using too high a flip angle $\beta$, signals that result from zero quantum coherence transfer pathways, COSY-type signals that result from coherence transfer between coupled spins, and signals from pathways that are enabled by strong coupling; and 3) extrinsic artefacts, e.g. chunking artefacts ${ }^{[21]}$, rapid pulsing artefacts, and digital filtration artefacts. One important feature of all the unwanted, extrinsic artefact, signals due to zero-quantum, COSY-type, and strong coupling coherence transfer pathways is that they all have phases that depend on the timing of the low flip angle pulses within the PSYCHE element. Unlike the pure shift signals of interest, which are fully refocused within the PSYCHE element, these signals acquire an extra phase shift that depends on the exact resonance frequencies of the spins involved. The presence of the field gradient during the PSYCHE element means that each horizontal slice of the sample has a different offset from resonance, so when acquiring the net signal of the whole sample all possible phases of these signals are averaged and cancellation results.

Figure 6 shows a schematic representation of the temporal and spatial mechanisms of signal averaging during a PSYCHE pulse sequence element. The combination of pulsed field gradients and chirp pulses results in the PSYCHE element acting as a spin filter, suppressing signals if their frequency changes during the element, as is the case for COSY-type coherence transfer from one spin to another, or if their phase depends on the time between the two $\beta$ pulses, as is the case for pathways that involve zero-quantum coherence during the storage time of the stimulated echo. In both cases the net signal will average to zero, by temporal averaging in the case of COSY and strong coupling responses (Figure 6a), and spatial averaging in the case of zero-quantum responses (Figure 6b).

\section{All together now: simultaneous decoupling and suppression of unwanted signals}

The central idea of the PSYCHE method is that it achieves two goals in one. It delivers decoupled signals, using low-flip angle pulses, and it rids the spectrum of unwanted responses, by spatiotemporal averaging across the sample. Of course, the efficiency of this filtration depends on experimental parameters such as the amplitude of the pulsed field gradient and the sweep-rate of the pulses. The degree of attenuation of the unwanted responses is inversely proportional to the duration of the pulses, e.g. in order to eliminate COSY from coupled spins with a small difference in chemical shift, a long pulse is needed, meaning a slow sweep rate for a given bandwidth. The same argument applies to the suppression of zero-quantum and strong coupling responses. Therefore, compromises need to be made between spectral purity and signal loss due to relaxation and diffusion during the PSYCHE element, just as compromises are needed in choosing the flip angle $\beta$. 

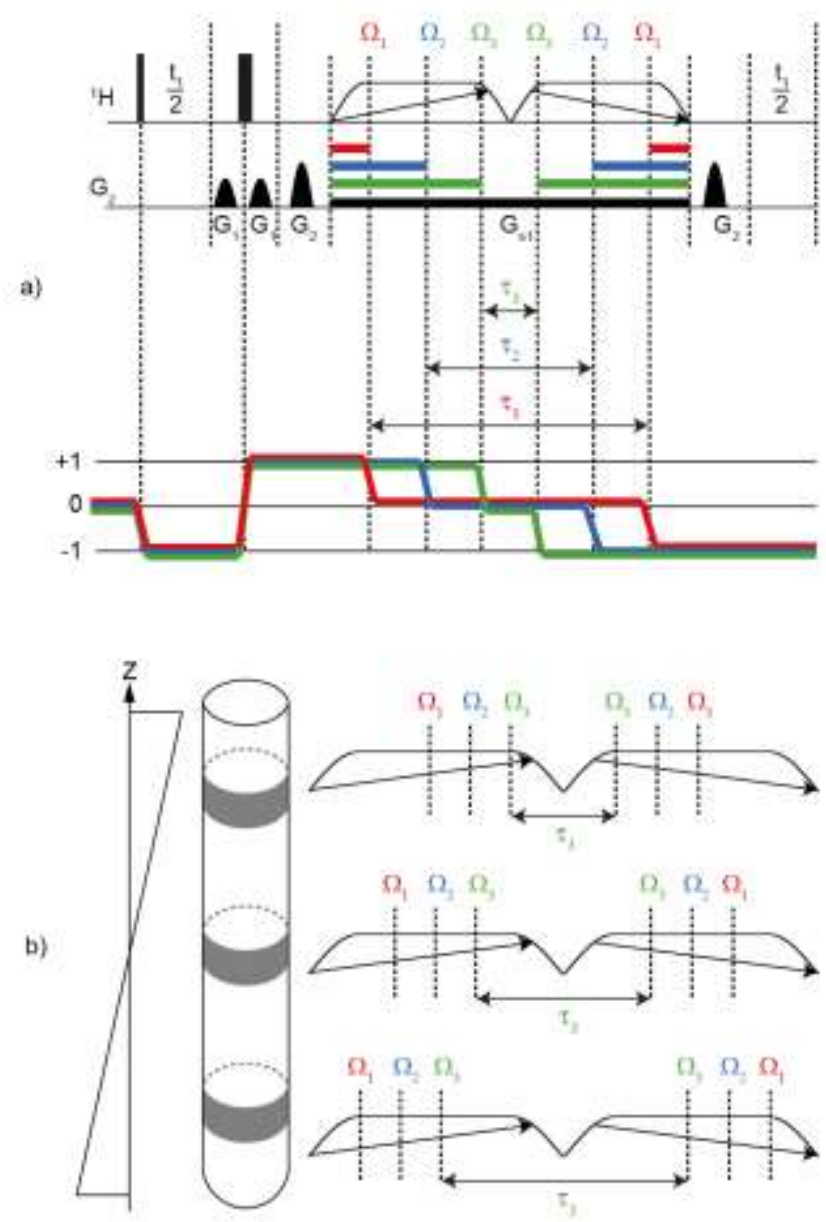

Figure 6. Illustration of spatio-temporal averaging in a PSYCHE pulse element. (a) The mechanism of temporal filtration is shown for three spins with different offsets $\left(\Omega_{1}, \Omega_{3}\right.$, and $\left.\Omega_{3}\right)$. The effect of the colour-coded gradient areas is to suppress any responses from signals that change frequency during the PSYCHE stimulated echo, since such signals will experience different gradient areas before and after the two $\beta$ pulses. The corresponding CTPs are shown below the pulse sequence. (b) The mechanism of spatial filtration is shown for the same resonance in three different parts of the sample. These experience different timings because the field gradient along the $z$-axis makes the resonant frequency depend on position. a)

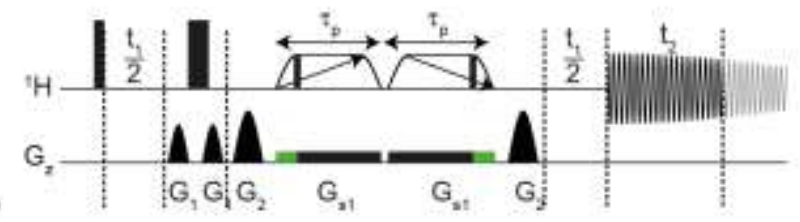

b)
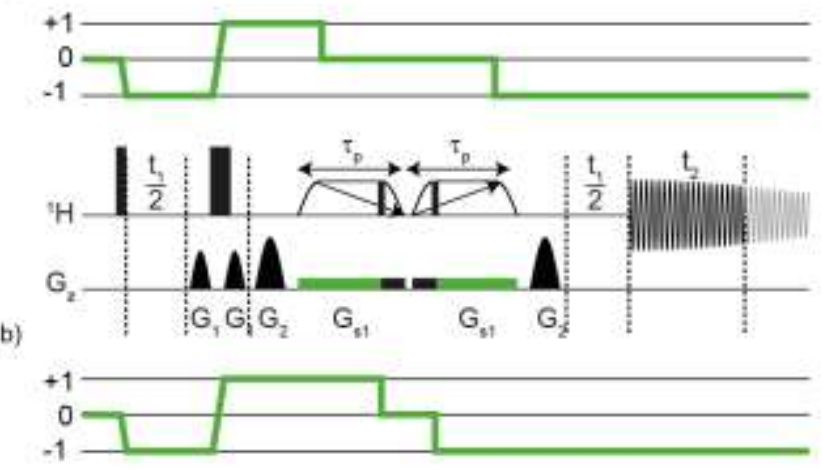

(a)

Figure 7. Diagrams showing how a combination of a pair of saltire chirp pulses (as shown in Figure 5) and pulsed field gradient results in selection of two CTPs at the same time and thus improves sensitivity compared to when unidirectional pairs are used. The green areas in (a) and (b) indicate the area of the encoding gradient which enforces CTPs shown below each sequence.

\section{Relationship between the PSYCHE and ZS methods}

In some practical respects the PSYCHE and Zangger-Sterk pulse elements have much in common: for example, a pulse sequence can be changed from one method to the other simply by changing the shape of the selective pulse waveform, leaving the rest of the sequence unchanged. However, there are important differences when choosing experimental parameters, which can cause some confusion when setting up these experiments. In the ZS method, each chemical shift (in the ideal case) is measured using a different slice of the sample, and the sensitivity of the method is dictated by the ratio between the selectivity of the frequencyselective pulse $\left(b w_{\text {sel }}\right)$ and the frequency range spanned by the encoding gradient $\left(s w_{g}=\gamma g L / 2 \pi\right)$, where $L$ is the height of the active volume of the sample. Therefore, the basic sensitivity loss is the ratio $b w_{s e l} / s w_{g}$, so for a broadband ( 10 ppm) $1 \mathrm{D}{ }^{1} \mathrm{H}$ pure shift spectrum on a $500 \mathrm{MHz}$ spectrometer, a frequency-selective pulse of $100 \mathrm{~Hz}$ bandwidth gives about $2 \%$ of the available sensitivity. Restricting observation to just part of a spectrum reduces $s w_{g}$ and improves sensitivity; the limiting case is the band-selective method where no gradient is used under the frequency-selective pulse and maximum signal is obtained. At first sight it is tempting to increase the bandwidth $b w_{\text {sel }}$ of the selective $180^{\circ}$ pulse, but spectral purity in a ZS experiment depends on the selectivity of this pulse. Clean results are only obtained if the bandwidth of the pulse is less than the smallest difference in Larmor frequency between coupled spin. In systems with coupled spins with similar chemical shifts this makes very selective pulses necessary, resulting in severe sensitivity loss not 
only from the ratio $b w_{s e l} / s w_{g}$ but also from relaxation, diffusion and convection.

In the PSYCHE method, all spins are irradiated in the whole sample, and it is the low flip angle pulses that are responsible for distinguishing between active and passive spins. The basic sensitivity penalty is now determined by the flip angle $\beta$, irrespective of the spin system parameters. In the ZS method, the experimenter has to choose whether to decouple a whole spectrum with poor sensitivity, or to decouple only part of the spectrum, or only coupled spins with a large chemical shift difference, with better sensitivity. The choice to be made with PSYCHE is between a very clean spectrum, with small recoupling artefacts but poorer sensitivity, and a less clean spectrum, with larger recoupling artefacts, but stronger signals. The important point here is that where signal-to-noise ratio is a problem, recoupling artefacts will not be: if the pure shift signals have a $S / N$ ratio of $20: 1$, it does not matter whether the recoupling artefacts are $1 \%$ or $5 \%$ of the parent signals, they will not be visible against the noise background. Of course, recoupling artefacts are not the only source of spectral impurities in PSYCHE, and as already noted the degree of suppression of intrinsic artefacts will depend both on the spin system and the experimental parameters. However, the only extra sensitivity penalty incurred by lengthening the PSYCHE element duration to improve the spatiotemporal averaging is that due to relaxation, diffusion, and convection ${ }^{[22]}$ during the long pulse elements, which will effect the PSYCHE and ZS methods to more or less the same extent.

PSYCHE is unusual among NMR techniques in requiring the experimenter to embrace the presence of intrinsic artefacts. Most NMR experiments are designed to give (near-) perfect results in perfect equipment, with only extrinsic artefacts in practical spectrometers. Thus the BS and ZS methods should ideally be capable of giving perfect results if used with perfect pulses in a perfect spectrometer. In the case of PSYCHE, the experimenter accepts the presence of the weak intrinsic recoupling artefacts and the limitations of spatiotemporal averaging as a price worth paying for the substantial sensitivity advantage it offers when measuring full-width pure shift spectra.

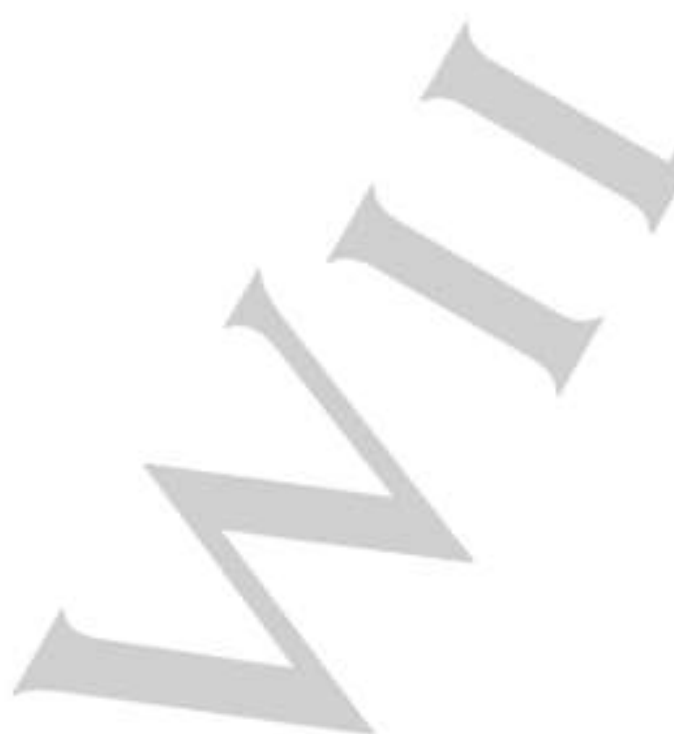

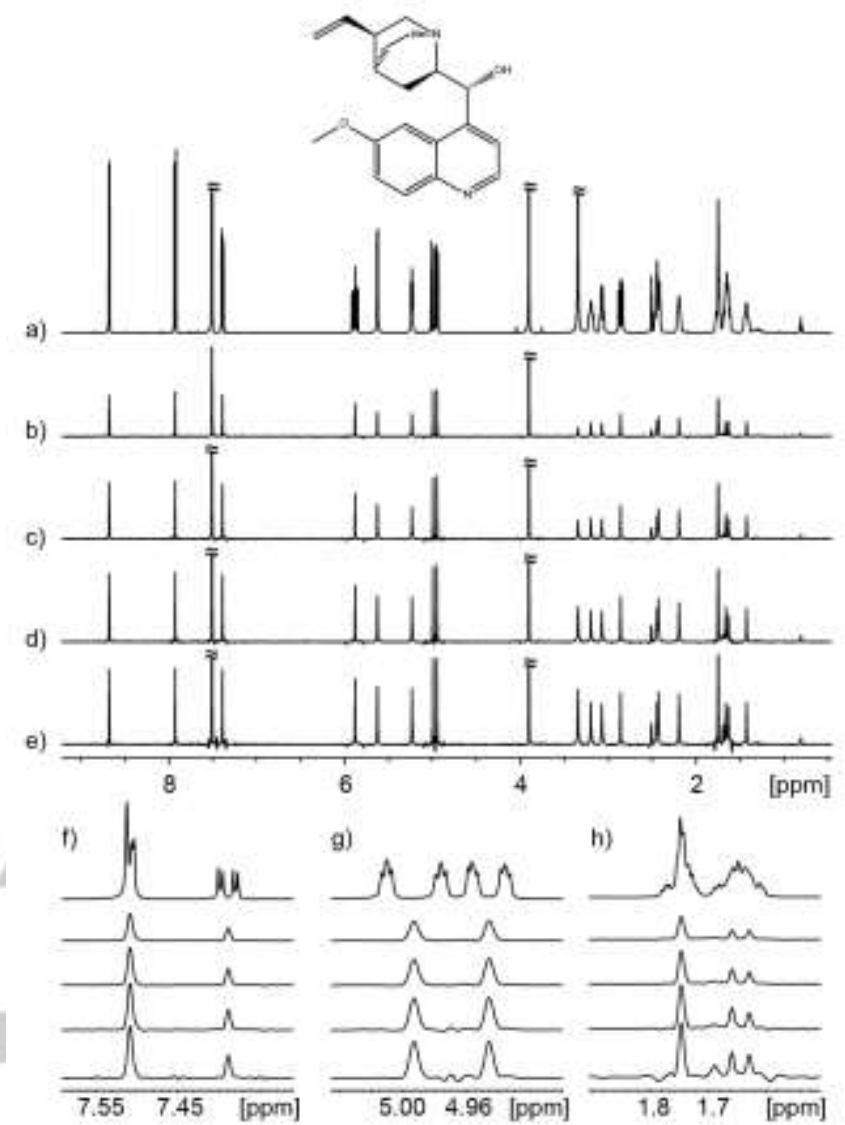

Figure 8. 1D $1 \mathrm{H}$ spectra of a $50 \mathrm{mM}$ sample of quinine (scheme) in DMSO-d6 acquired with (a) conventional pulse-acquire sequence and (b-e) 1D PSYCHE pulse sequence in which the total durations of PSYCHE pulse element were set to $30,60,90$, and $120 \mathrm{~ms}$ respectively and the sweep-width of $10 \mathrm{kHz}$ for all pulses, which correspond to sweep rates of $333,167,111$, and $83 \mathrm{kHz} / \mathrm{s}$ respectively.

\section{PSYCHE-based experiments}

Since the first publication of the PSYCHE method in $2014,,^{[5]}$ a variety of experiments using the PSYCHE pulse sequence element have been introduced, including $1 \mathrm{D}^{1} \mathrm{H} \mathrm{NMR}^{[5,23]}, 2 \mathrm{D}^{1} \mathrm{H}-$ ${ }^{1} \mathrm{H}$ TOCSY ${ }^{[24]}$ and variants using band-selective excitation ${ }^{[25]}$ and non-uniform sampling ${ }^{[26]}$, 2D J-resolved spectroscopy with absorption mode lineshapes (PSYCHE 2DJ[23] and RASA2DJ[27]), $z$-filtered 2DJ spectroscopy with interferogram acquisition ${ }^{[28]}$, the PSYCHEDELIC ${ }^{[29]}$ method for selective measurements of individual couplings, 1D ${ }^{1} \mathrm{H}$ NMR combined with selective TOCSY[30], PSYCHE-EASY-ROESY[31] for direct observation of intermolecular NOEs in crowded spectra, PSYCHE-iDOSY ${ }^{[32]}$ for mixture analysis, PSYCHE CPMG-HSQMBC ${ }^{[33]}$ for accurate measurements of heteronuclear couplings, ultrafast multi-slice selective J-resolved spectroscopy ${ }^{[34]}$, and Inrec-PSYCHE and CPMG-PSYCHE ${ }^{[35]}$ for $T_{1}$ and $T_{2}$ measurements. Here we discuss a few of these methods and introduce some current improvements in the application and implementation of the PSYCHE method. 
a)

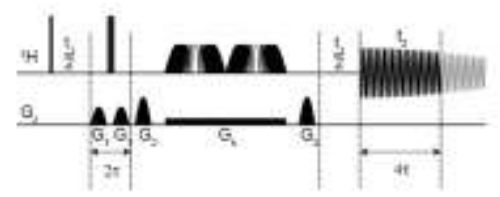

b)

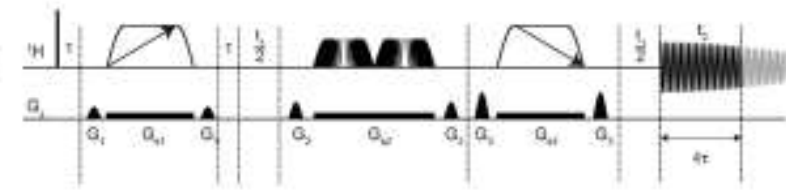

c)
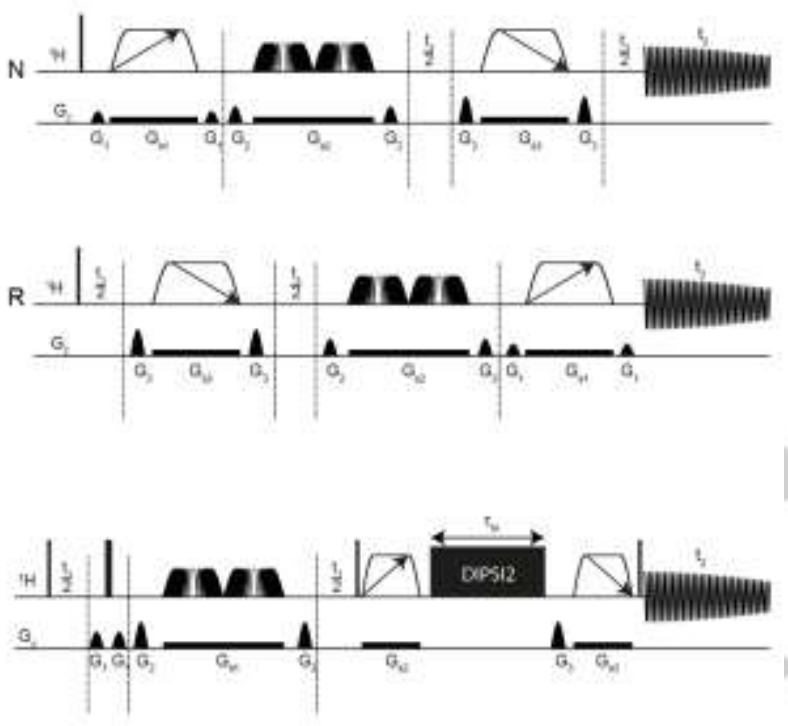

e)

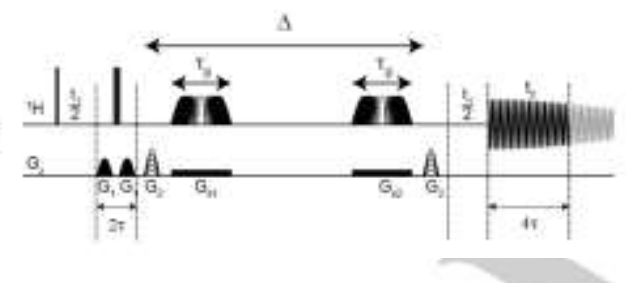

Figure 9. PSYCHE-based pulse sequences. (a) 1D PSYCHE with pseudo 2D interferogram acquisition, (b) 1D TSE-PSYCHE with pseudo 2D interferogram acquisition, (c) PSYCHE 2D J with sequences acquiring $\mathrm{N}$-type and R-type dataset for echo/anti echo acquisition scheme, (d) 2D F $F_{1}$-PSYCHE-TOCSY, and (e) PSYCHE-IDOSY. In all sequences the narrow and wide rectangles denote hard $90^{\circ}$ and $180^{\circ}$ RF pulses, the open trapezoid shapes with arrow denote $180^{\circ}$ unidirectional chirp pulses, and the amplitude modulated trapezoids are low flip angle saltire chirp pulses. All filled half-sine shapes $\left(G_{1}, G_{2}\right.$, etc.) indicate field gradient pulses for coherence transfer pathway (CTP) selection. Incremented open half-sine shapes $\left(G_{2}\right)$ in (e) indicate diffusion-encoding gradient pulses with variable gradient amplitudes. All rectangular gradient pulses $\left(G s_{1}, G s_{2}\right.$, etc.) are weak gradients applied during the swept-frequency (saltire and unidirectional chirp) pulses. Delay $t_{1}$ indicates the incremented evolution time in $2 \mathrm{D}$ and pseudo-2D experiments. The delay $\mathrm{\tau}$ is set to the value $1 / 4 s w_{1}$ for interferogram acquisition schemes. $T_{m}$ in $(d)$ is the duration of the mixing sequence (DIPSI2), and $\Delta$ and $I_{\mathrm{p}}$ in (e) are diffusion delay and the duration of one saltire chirp pulse respectively.

\section{Improved spectral purity: 1D Triple Spin Echo PSYCHE and PSYCHE 2DJ}

As we have seen in the discussion of the mechanism of the PSYCHE method, one problem with suppression of unwanted signals using spatio-temporal averaging in general, and in PSYCHE, in particular, is that the achievable attenuation is inversely proportional to the frequency of oscillation to be suppressed, i.e. the slower the time-evolution of unwanted coherences the more difficult it is to introduce enough phase variation for them to cancel. To translate this into the context of chemical shift and scalar couplings of interacting spins, the closer coupled spins are in chemical shift, the more difficult it is to achieve efficient spatio-temporal averaging. An extreme case of this is strong coupling between spins, when the difference in chemical shift between coupled spins is comparable with the magnitude of scalar coupling. Additional spatio-tempora averaging can be achieved either by lengthening the PSYCHE pulse element (i.e. using a slower sweep-rate), as discussed in detail below, or, more efficiently, by replacing the hard $180^{\circ}$ pulse with two extra swept-frequency chirp pulses in the presence of weak pulsed field gradients, as used previously by Thrippleton et al. for attenuating strong coupling responses ${ }^{[20]}$. The triple spin echo (TSE) experiment that results offers a major improvement in performance over the parent 1D PSYCHE experiment, as shown in Figure $\mathbf{1 0}$ and Figure 11. TSE-PSYCHE typically gives much cleaner spectra, but any extra diffusion, convection, or relaxation during the longer pulse sequence will reduce the signal-to-noise ratio compared to the parent PSYCHE experiment. The TSE approach is also very tolerant of $B_{1}$ field inhomogeneity and pulse miscalibration (Figure 12), giving very good results even for RF power errors of up to $\pm 3 \mathrm{~dB}$ (which would correspond to ca. $\pm 60^{\circ}$ errors in a hard $180^{\circ}$ pulse). This sequence configuration also lends itself perfectly to the elegant acquisition scheme for $2 \mathrm{D} \mathrm{J}$ resolved spectroscopy first proposed by Pell and Keeler ${ }^{[10]}$ to solve the problem of phase-twist lineshapes in the classic 2D J (Jresolved) spectroscopy ${ }^{[2]}$. The TSE-PSYCHE 2DJ sequence results in virtually artefact-free absorption-mode 2D J spectra (Figure 13), with little need for any special post-processing such as symmetrisation ${ }^{[20]}$. This approach can be used both for producing pure shift $1 \mathrm{D}$ spectra, by projecting the $2 \mathrm{D}$ spectrum at $45^{\circ}$ in frequency space, and for extracting high-resolution multiplet structures. The method approaches the ideal limit of resolution of a ${ }^{1} \mathrm{H}$ NMR spectrum, since as a result of $B_{0}$ inhomogeneity being refocused the linewidths in $F_{1}$ are very close to the natural linewidths dictated by $T_{2}$. 

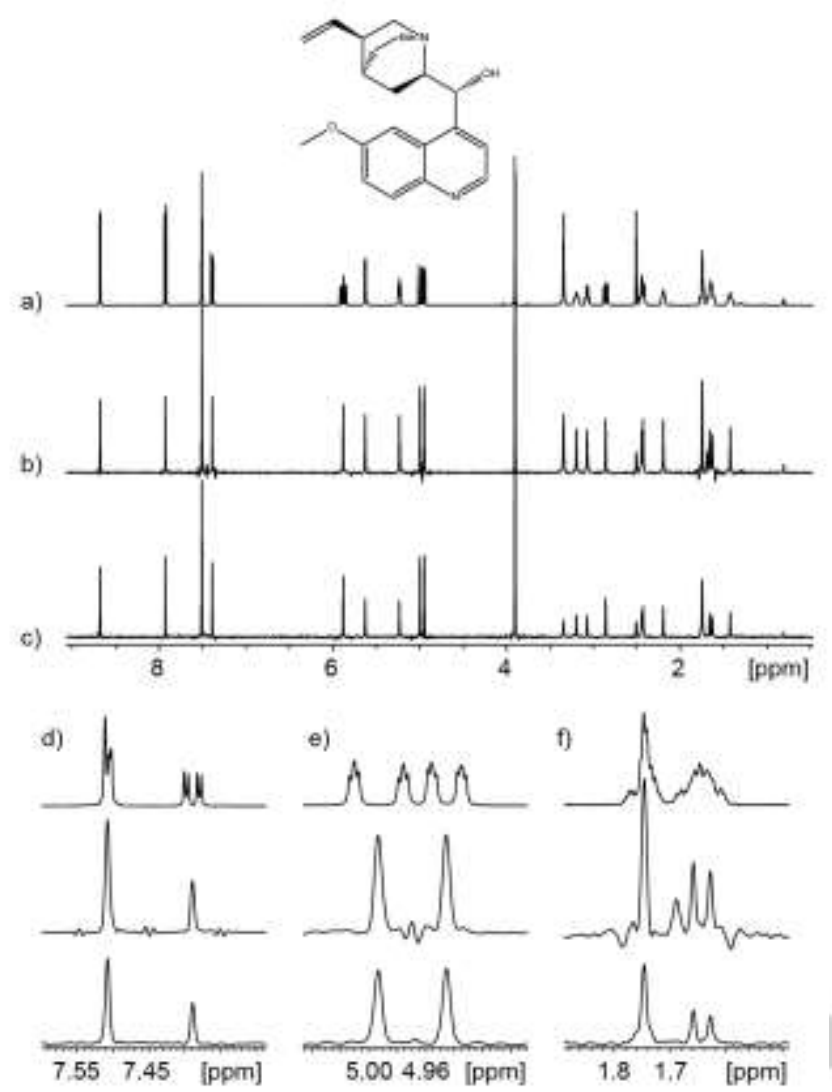

Figure 10. 1D ${ }^{1} \mathrm{H}$ spectra of a $50 \mathrm{mM}$ sample of quinine (structure at top) in DMSO- $d_{6}$ acquired with (a) conventional pulse-acquire sequence, (b) 1D PSYCHE, and (c) 1D TSE-PSYCHE. Expansions in (d-f) show regions involving strong coupling.
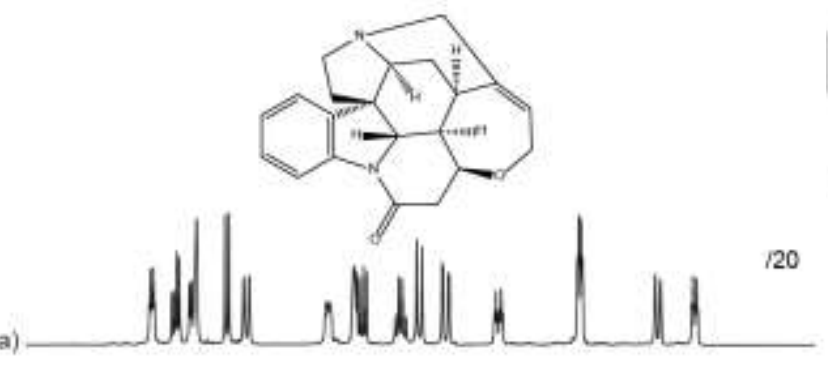

120

b)
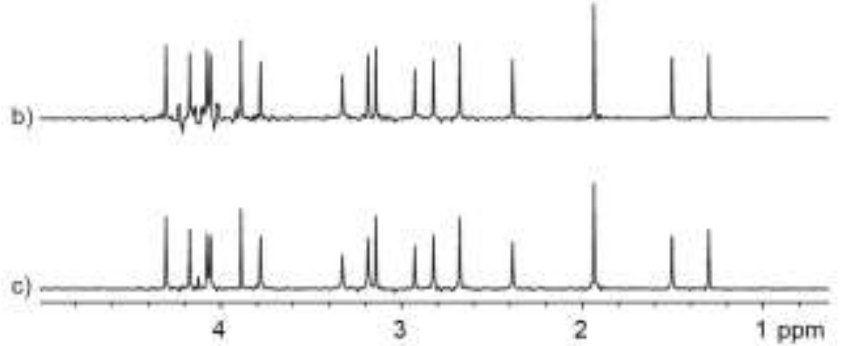

Figure 11. Spectra of a sample of strychnine (structure at top) in chloroform-d acquired by (a) conventional 1D ${ }^{1} \mathrm{H}$, (b) 1D PSYCHE, and (c) 1D TSE-PSYCHE. In (b) and (c) the flip angle $\beta$ of the PSYCHE pulse element was set to $15^{\circ}$. The improvement provided by the additional spatio-temporal averaging in the TSE experiment can be clearly seen in the region around $4 \mathrm{ppm}$.

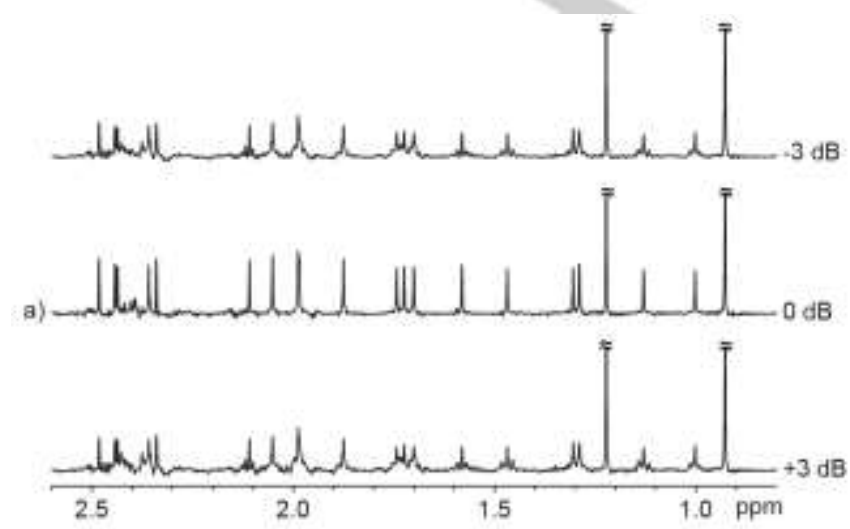

b

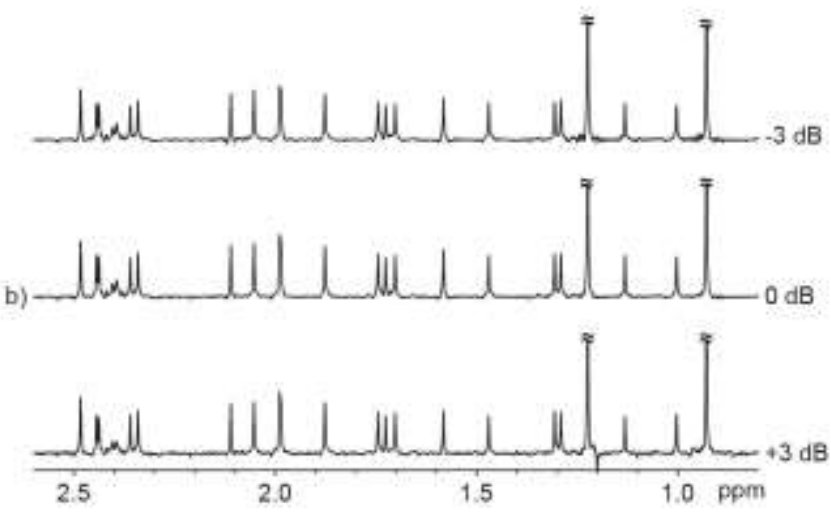

Figure 12. The effect of variation of $B_{1}$ field amplitude on (a) 1D PSYCHE, and (b) 1D TSE-PSYCHE for a sample of androst-4-ene-3,17-dione in chloroform-d. The values on the right side of each spectrum indicate the deviation of the RF power used from its optimum value. The TSE-PSYCHE experiment shows very good tolerance of pulse miscalibration. 


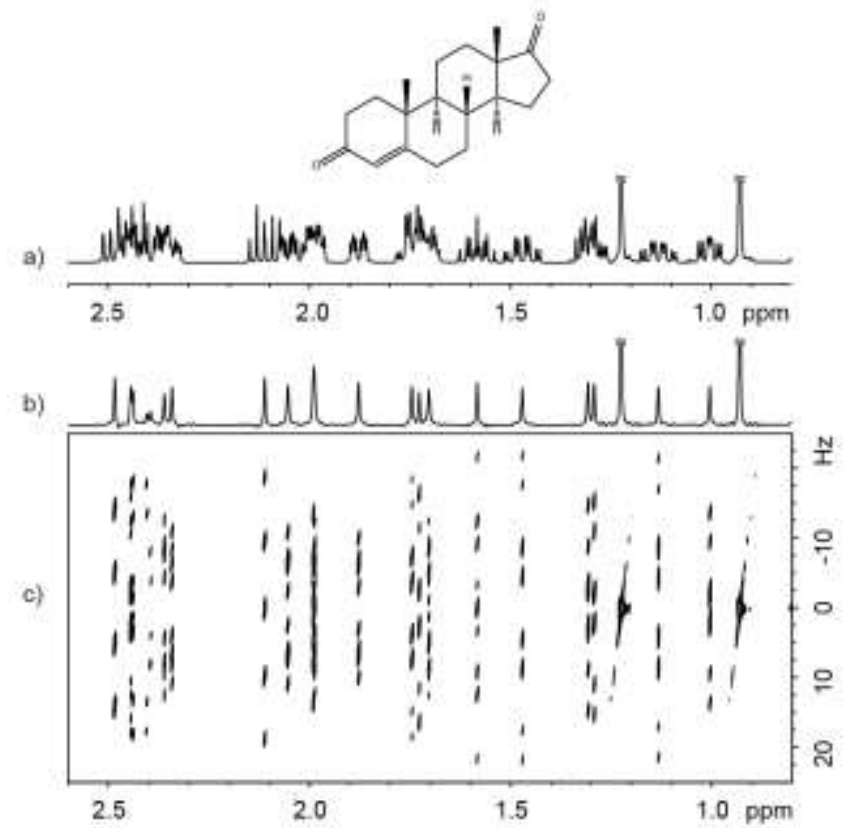

Figure 13. Spectra of androst-4-ene-3,17-dione (structure at top) in chloroform$d$ : (a) conventional pulse-acquire ${ }^{1} \mathrm{H}$ spectrum, (c) $45^{\circ}$ tilted absorption mode $2 \mathrm{D}$ $\mathrm{J}$ spectrum acquired with the PSYCHE 2D J pulse sequence. (b) shows the pure shift spectrum obtained by projection of the tilted $2 \mathrm{D} \mathrm{J}$ spectrum onto $F_{2}$.

\section{Decoupling in the indirect dimension: $\boldsymbol{F}_{1-}$ PSYCHE-TOCSY}

The great majority of experiments to date that use broadband homonuclear decoupling in an indirect dimension, many of them in protein NMR, use Bax's constant-time method ${ }^{[36]}$. This has the severe disadvantage of giving signal intensities that are strongly modulated, depending critically on the choice of constant time. An indirect domain method that avoids this problem, the timereversal method, was reported quite early on ${ }^{[37]}$ but has not found significant application. A variety of other methods have subsequently been described that use J-refocusing pulse sequence elements in the middle of the $t_{1}$ evolution time, to give decoupling in the $F_{1}$ dimension. Examples of $F_{1}$ pure shift 2D methods include constant-time COSY[9], $F_{1}$-ZS-HSQC ${ }^{[17]}$, and $F_{1}$ BS-TOCSY[38].

The first application of PSYCHE in 2D NMR was in the indirect dimension of a $2 \mathrm{D}^{1} \mathrm{H}-{ }^{-1} \mathrm{H}$ TOCSY ${ }^{[39]}$ experiment. Decoupling in the $F_{1}$ rather than in the $F_{2}$ dimension, as in the earliest ZS pure shift $2 \mathrm{D}$ experiments ${ }^{[8 a]}$, has the advantages that standard FT-based processing can be used, and that it is immune to the chunking artefacts seen in interferogram acquisition. Although $F_{1^{-}}$ decoupling requires higher digital resolution along this dimension than in the $F_{2}$-ZS-TOCSY experiment, ${ }^{[8 a]}$ this incurs no additional time penalty since the latter requires multiple data chunks to be acquired for each $t_{1}$ increment. In addition, using conventional acquisition in $t_{2}$ allows high-resolution multiplet structure to be measured in $F_{2}$ at no extra cost in acquisition time, which combined with chemical-shift only signal dispersion in $F_{1}$ gives easily interpretable spectra. If indirect covariance data processing ${ }^{[40]}$ is used, the same experimental data yield an ultrahigh-resolution 2D correlation spectrum (Figure 14c) which is pure shift in both dimensions.
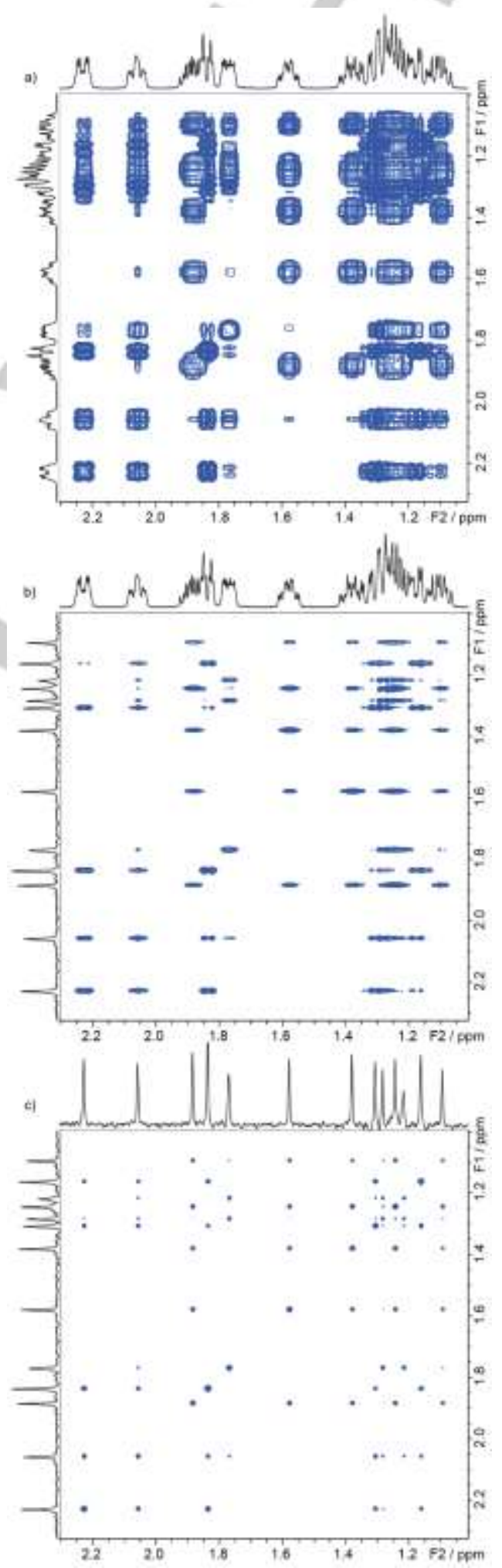
Figure 14. Spectra of a sample of estradiol in DMSO-d6 (a) acquired with the conventional TOCSY sequence, (b) acquired with $F_{1}$-PSYCHE-TOCSY, and (c) double pure shift TOCSY spectrum obtained by applying indirect covariance processing to spectrum (b).

\section{Mixture analysis: PSYCHE-iDOSY}

Diffusion-ordered spectroscopy (DOSY) ${ }^{[41]}$ was one of the first experiments to use pure shift NMR, in the form of ZS-PFGSTE DOSY ${ }^{[7]}$. The reason for this is the crucial importance in DOSY of avoiding overlap between different signals in the direct dimension. This is because DOSY relies on nonlinear least squares fitting to the Stejskal-Tanner equation ${ }^{[42]}$ to determine the positions of signals in the diffusion domain. If signals with different diffusion coefficients overlap, mono-exponential fitting will result in a single, erroneous, compromise diffusion coefficient. ${ }^{[41 c, 43]}$ Pure shift DOSY experiments are therefore very desirable, even at substantial cost in sensitivity, because they drastically reduce the incidence of peak overlap in spectra with extensive homonuclear multiplet structure.

As explained earlier, the PSYCHE pulse sequence element is related to the stimulated echo, one of the earliest pulse sequences used for the measurement of diffusion ${ }^{[44]}$. In the PSYCHE-iDOSY ${ }^{[32]}$ experiment the PSYCHE pulse sequence element plays a dual role, both refocusing the effects of scalar couplings and encoding the effects of diffusion, in the form of signal attenuation. Here the $i$ in PSYCHE -iDOSY indicates that the diffusion-encoding part of the sequence is internal to the PSYCHE pulse element. ${ }^{[45]}$ There are two advantages to internal diffusion encoding, as opposed to concatenating a pure shift sequence (e.g. interferogram PSYCHE) with a diffusion-encoding sequence (e.g. Oneshot DOSY ${ }^{[46]}$ : firstly, a twofold improvement in sensitivity can be achieved by avoiding the need for an additional stimulated echo, and secondly, a much simpler pulse sequence results, which suffers much less from pulse imperfections and hence needs far less phase cycling for clean results.

Figure 15 shows the results of applying the PSYCHE-iDOSY sequence and a conventional Oneshot pulse sequence ${ }^{[46 \mathrm{~b}]}$ to a mixture of the isomers vitamin $D_{3}$ and provitamin $D_{3}$. The significantly better statistics of the fitting for the diffusional decay in the PSYCHE-iDOSY experiment make it possible to distinguish the signals of the two molecules despite their having very similar diffusion coefficients. One slight complication is that the spatiotemporal encoding used in PSYCHE makes the parametrization of the Stejskal-Tanner equation ${ }^{[42]}$ vary with position in the sample. Fortunately the signal attenuation for the total sample, integrated over the active volume, is very well approximated by the usual Gaussian Stejskal-Tanner equation with a small constant correction applied to the diffusion-encoding gradient amplitude. It is thus possible to use standard processing software (the DOSY Toolbox, ${ }^{[47]}$ Bruker Dynamics Center, VnmrJ etc.) simply by adding a small constant, calculable from the experimental parameters, to the list of gradient amplitudes. The full derivation of the modified Stejskal-Tanner equation can be found in the supporting material of reference ${ }^{[32]}$.
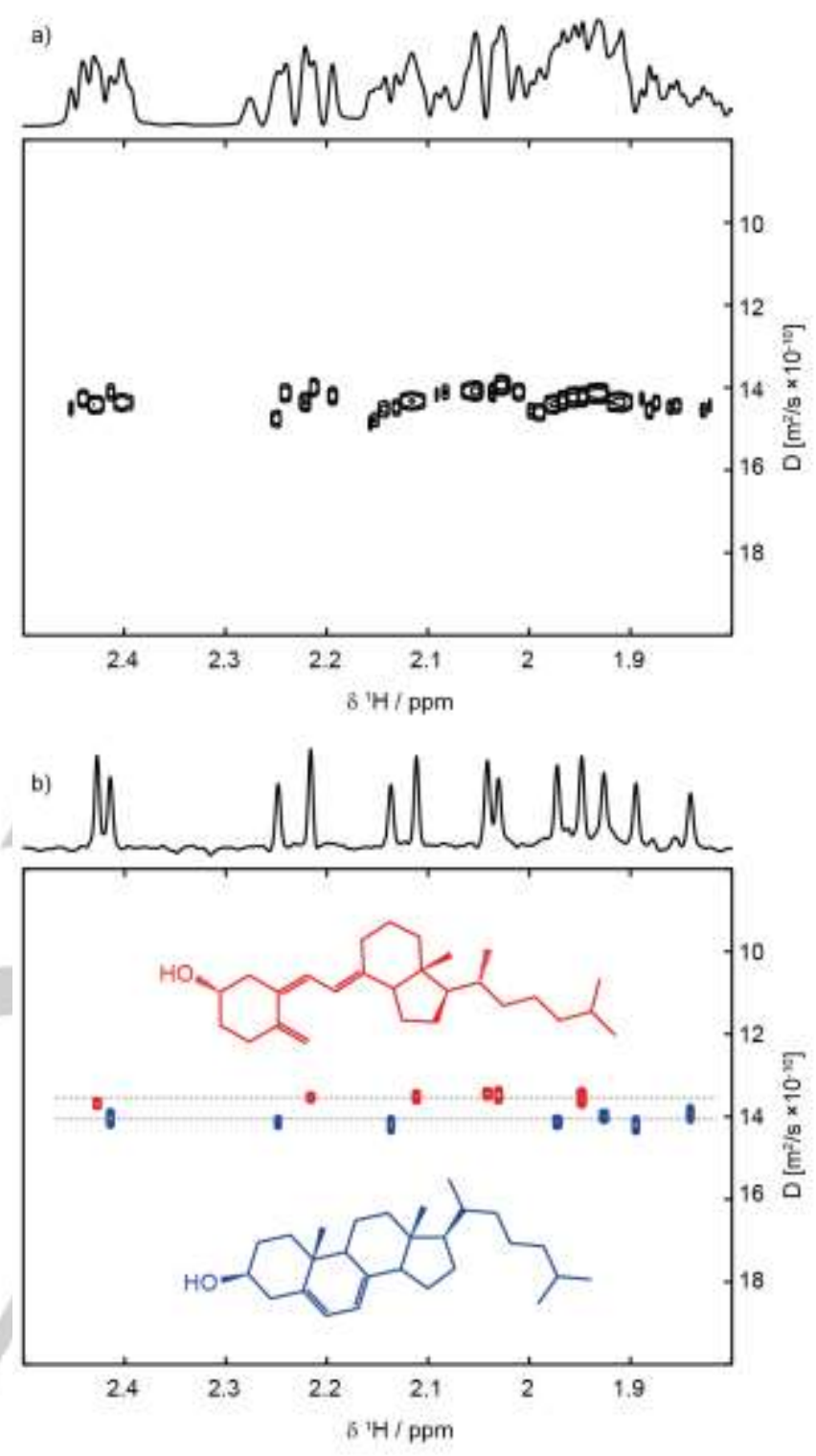

Figure 15. Expanded regions from DOSY spectra of a mixture of vitamin $D_{3}$ (scheme in red) and provitamin $\mathrm{D}_{3}$ (scheme in blue), $50 \mathrm{mM}$ each, in acetone$d_{6}$, acquired with (a) Oneshot DOSY[46b] and (b) PSYCHE-iDOSY pulse sequences. Both datasets were processed using the DOSY Toolbox.. ${ }^{[4]}$

\section{General remarks and conclusion}

In this review we have presented some notes on the mechanics and practicalities of the PSYCHE method. The effects and importance of experimental parameters such as flip angle, sweep rate, etc. have been highlighted, and the use of saltire pulses to improve the trade-off between sensitivity and spectral purity has been described, and finally some examples of the use of the PSYCHE element in more complex 1D and 2D experiments have been illustrated. A more complete description of the theory of the PSYCHE pulse sequence element will be presented elsewhere. 


\section{Experimental Section}

All experimental spectra were recorded at a nominal temperature of $298 \mathrm{~K}$ on a Bruker Avance II+ $500 \mathrm{MHz}$ spectrometer, with a $5 \mathrm{~mm}$ BBO probe equipped with a $z$-gradient coil with a maximum nominal gradient strength of $53.5 \mathrm{G} \mathrm{cm}^{-1}$.

For 1D PSYCHE experiment (Figure 9a, Figure 10b, and Figure 11b), $G_{1}$ and $G_{2}$ are half-sine gradient pulses, used for selection of the desired coherence transfer pathways, and have amplitudes of $26 \mathrm{G} \mathrm{cm}^{-1}$ and $40 \mathrm{G}$ $\mathrm{cm}^{-1}$ respectively, and a duration of $1 \mathrm{~ms}$ each. $G_{\mathrm{s}}$ is a rectangular gradient pulse, aligned with the midpoint of the pair of chirp pulses, and has an amplitude of $1.5 \mathrm{G} \mathrm{cm}^{-1}$.

For 1D TSE-PSYCHE (Figure 9b, Figure 10c, and Figure 11c), and PSYCHE-2D J (Figure 9c and Figure 13) experiments, $G_{1}, G_{2}$ and $G_{3}$ are half-sine gradient pulses, used for selection of the desired coherence transfer pathways, and have amplitudes of $18 \mathrm{G} \mathrm{cm}^{-1}, 26 \mathrm{G} \mathrm{cm}^{-1}$ and $40 \mathrm{G}$ $\mathrm{cm}^{-1}$ respectively, and a duration of $1 \mathrm{~ms}$ each. $G_{\mathrm{s} 2}$ is a rectangular gradient pulse, aligned with the midpoint of the pair of chirp pulses, and has an amplitude of $1.5 \mathrm{G} \mathrm{cm}^{-1} . G_{\mathrm{s} 1}$ and $G_{\mathrm{s} 3}$ are rectangular gradient pulses, applied to dephase coherences that arise from strong couplings, and have an amplitude of $1.0 \mathrm{G} \mathrm{cm}^{-1}$. The two $180^{\circ}$ chirp pulses have a duration of $40 \mathrm{~ms}$, a bandwidth of $10 \mathrm{kHz}$, and an RF amplitude of $447 \mathrm{~Hz}$ each. The PSYCHE pulse sequence element consists of two low flip angle saltire chirp pulses with flip angle of $15^{\circ}$, an r.f. amplitude of $50 \mathrm{~Hz}$, a duration of $15 \mathrm{~ms}$, and a bandwidth of $10 \mathrm{kHz}$ each. The 1D TSE-PSYCHE experiment was run with the same parameters as for PSYCHE-2D J, data were processed using a reconstruction macro.

For $F_{1}$-PSYCHE-TOCSY experiment (Figure $9 \mathbf{d}$ and Figure 14), $G_{1}, G_{2}$, $G_{3}$ are field gradient pulses with half sine shapes. Gradient pulses $G_{1}$ and $G_{2}$ are used for selection of desired coherence transfer pathways (CTP) and had amplitudes of $24 \mathrm{G} \mathrm{cm}-1$ and $38 \mathrm{G} \mathrm{cm}^{-1}$ respectively and duration of $1 \mathrm{~ms}$ each. Gradient pulse $G_{\mathrm{s} 1}$ is aligned with the center of the two chirp pulses and had an amplitude of $1.5 \mathrm{G} \mathrm{cm}^{-1}$. Gradient pulses $G_{\mathrm{s} 2}$ and $G_{\mathrm{s} 3}$ are applied to dephase zero quantum coherences and had an amplitude of $2 \mathrm{G} \mathrm{cm}-1$. Gradient pulse $G_{3}$ is a spoil pulse and had an amplitude of 12 $\mathrm{G} \mathrm{cm}^{-1}$ and a duration of $1 \mathrm{~ms}$. The number of transients was set to 8, TD and TD1 were set to 2048, and SW and SW1 were set to $5000 \mathrm{~Hz}$, in a total experiment time of $9 \mathrm{~h}$.

For PSYCHE-iDOSY experiment (Figure 9e and Figure 15) 12 gradient strengths ranging from 2.7 to $34.8 \mathrm{G} \mathrm{cm}^{-1}$ in equal steps of gradient squared were used for the diffusion-encoding gradient $G_{2}$. A diffusion delay $\Delta$ of $0.1 \mathrm{~s}$ was used, with a diffusion-encoding pulse width of $1.5 \mathrm{~ms}$. $\mathrm{G}_{1}$ was a half-sine gradient pulse of $41.2 \mathrm{G} \mathrm{cm}^{-1}$ with a duration of $1 \mathrm{~ms}$, used for CTP selection. The PSYCHE pulse sequence element consists of two saltire chirp pulses of flip angle $20^{\circ}$, with an r.f. amplitude of $64 \mathrm{~Hz}$, a duration of $15 \mathrm{~ms}$, and a bandwidth of $10 \mathrm{kHz}$. In the pure shift dimension of the PSYCHE-iDOSY experiment, 20 increments of $t_{1}$ were used.

In all experiments, except $F_{1}$-PSYCHE-TOCSY, the spectral window in the direct dimension was set to $10000 \mathrm{~Hz}$, and the size of the FID to $8 \mathrm{k}$ complex points. In pure shift experiments, the spectral window in the interferogram (indirect) dimension was set to $50 \mathrm{~Hz}$, corresponding to a 20 ms duration for each chunk of data in the pure shift FID.

\section{Acknowledgements}

This work was funded by the Engineering and Physical Sciences Research Council (grant numbers EP/L018500 and EP/M013820).

Keywords: NMR spectroscopy • Analytical methods • PSYCHE - Pure shift NMR $\bullet$ Homonuclear decoupling 


\section{WILEY-VCH}

\section{CONCEPT}

This review offers some insights into theoretical and practical aspects of the PSYCHE (Pure Shift Yielded by Chirp Excitation) method, and into the effects and importance of the experimental parameters, to present some recent improvements.

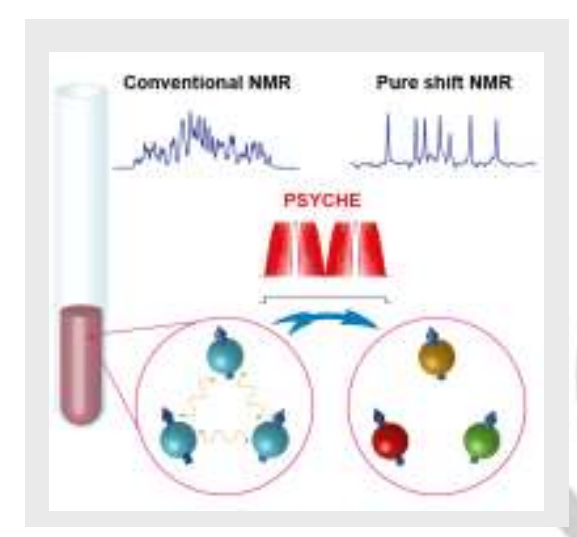

Mohammadali Foroozandeh, Gareth A. Morris, and Mathias Nilsson*

Page No. - Page No.

PSYCHE Pure Shift NMR Spectroscopy

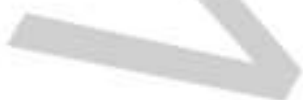

a) R. W. Adams, in eMagRes, John Wiley \& Sons, Ltd, 2014; b) L. Castañar, T. Parella, Magn. Reson. Chem. 2015, 53, 399-426; c) K. Zangger, Prog. Nucl. Magn. Reson. Spectrosc. 2015, 86-87, 1-20.

W. P. Aue, J. Karhan, R. R. Ernst, J. Chem. Phys. 1976, 64, 4226-4227.

K. Zangger, H. Sterk, J. Magn. Reson. 1997, 124, 486-489.

J. R. Garbow, D. P. Weitekamp, A. Pines, Chem. Phys. Lett. 1982, 93, 504-509.

M. Foroozandeh, R. W. Adams, N. J. Meharry, D. Jeannerat, M. Nilsson, G. A. Morris, Angew. Chem. Int. Ed. 2014, 53, 6990-6992. a) J. A. Aguilar, S. Faulkner, M. Nilsson, G. A. Morris, Angew. Chem. Int. Ed. 2010, 49, 3901-3903; b) N. Giraud, L. Béguin, J. Courtieu, D. Merlet, Angew. Chem. Int. Ed. 2010, 49, 3481-3484; c) N. H. Meyer, K. Zangger, Angew. Chem. Int. Ed. 2013, 52, 7143-7146. M. Nilsson, G. A. Morris, Chem. Commun. 2007, 933-935.

a) G. A. Morris, J. A. Aguilar, R. Evans, S. Haiber, M. Nilsson, J. Am. Chem. Soc. 2010, 132, 12770-12772; b) A. Cotte, M. Foroozandeh, D. Jeannerat, CHIMIA 2012, 66, 764-769.

[9] J. A. Aguilar, A. A. Colbourne, J. Cassani, M. Nilsson, G. A. Morris, Angew. Chem. Int. Ed. 2012, 51, 6460-6463.

[10] A. J. Pell, J. Keeler, J. Magn. Reson. 2007, 189, 293-299.

a) R. W. Adams, L. Byrne, P. Kiraly, M. Foroozandeh, L. Paudel, M. Nilsson, J. Clayden, G. A. Morris, Chem. Commun. 2014, 50, 2512-2514; b) L. Castañar, P. Nolis, A. Virgili, T. Parella, Chem. Eur. J. 2013, 19, 17283-17286; c) J. Ying, J. Roche, A. Bax, J. Magn. Reson. 2014, 241, 97-102.

a) J. A. Aguilar, M. Nilsson, G. A. Morris, Angew. Chem. Int. Ed. 2011, 50, 9716-9717; b) A. Lupulescu, G. L. Olsen, L. Frydman, J. Magn. Reson. 2012, 218, 141-146.

[13] a) L. Paudel, R. W. Adams, P. Király, J. A. Aguilar, M. Foroozandeh, M. J. Cliff, M. Nilsson, P. Sándor, J. P. Waltho, G. A. Morris, Angew. Chem. Int. Ed. 2013, 52, 11616-11619; b) P. Sakhaii, B. Haase, W. Bermel, J. Magn. Reson. 2009, 199, $192-198$. P. Kiraly, R. Adams, L. Paudel, M. Foroozandeh, J. Aguilar, I. Timári, M. Cliff, M. Nilsson, P. Sándor, G. Batta, J. Waltho, K. Kövér, G. Morris, J. Biomol. NMR 2015, 62, 43-52.

A. Bax, A. F. Mehlkopf, J. Smidt, J. Magn. Reson. 1979, 35, 167-169.

[16] A. Bax, J. Magn. Reson. 1983, 53, 517-520.

a) M. Foroozandeh, P. Giraudeau, D. Jeannerat, ChemPhysChem 2011, 12, 2409-2411; b) M. Foroozandeh, P. Giraudeau, D. Jeannerat, Magn. Reson. Chem. 2013, 51, 808-814.

a) H. Oschkinat, A. Pastore, P. Pfändler, G. Bodenhausen, J. Magn. Reson. 1986, 69, 559-566; b) A. J. Pell, R. A. E. Edden, J. Keeler, Magn. Reson. Chem. 2007, 45, 296-316.

M. J. Thrippleton, R. A. E. Edden, J. Keeler, J. Magn. Reson. 2005, 174, 97-109.

P. Moutzouri, Y. Chen, M. Foroozandeh, P. Kiraly, A. R. Phillips, S. R. Coombes, M. Nilsson, G. A. Morris, Chem. Commun. 2017, 53, 1018810191.

M. Foroozandeh, R. W. Adams, M. Nilsson, G. A. Morris, J. Am. Chem. Soc. 2014, 136, 11867-11869.

V. M. R. Kakita, S. P. B. Vemulapalli, J. Bharatam, Magn. Reson. Chem. 2016, 54, 308-314.

V. M. R. Kakita, R. V. Hosur, ChemPhysChem 2016, 2304-2308.

Y. Huang, Y. Yang, S. Cai, Z. Chen, H. Zhan, C. Li, C. Tan, Z. Chen, Anal. Chem. 2017, 89, 12646-12651.

P. Kiraly, M. Foroozandeh, M. Nilsson, G. A. Morris, Chem. Phys. Lett. 2017, 683, 398-403.

D. Sinnaeve, M. Foroozandeh, M. Nilsson, G. A. Morris, Angew. Chem. Int. Ed. 2016, 55, 1090-1093. 


\section{WILEY-VCH}

\section{CONCEPT}

[30] a) G. D. Poggetto, L. Castañar, G. A. Morris, M. Nilsson, RSC Advances 2016, 6, 100063-100066; b) V. M. R. Kakita, R. V. Hosur, ChemPhysChem 2016, 17, 4037-4042.

[31] E. Procházková, A. Kolmer, J. Ilgen, M. Schwab, L. Kaltschnee, M. Fredersdorf, V. Schmidts, R. C. Wende, P. R. Schreiner, C. M. Thiele, Angew. Chem. Int. Ed. 2016, 55, 15754-15759.

M. Foroozandeh, L. Castañar, L. G. Martins, D. Sinnaeve, G. D. Poggetto, C. F. Tormena, R. W. Adams, G. A. Morris, M. Nilsson, Angew. Chem. Int. Ed. 2016, 55, 15579-15582.

[33] I. Timári, L. Szilágyi, K. E. Kövér, Chemistry - A European Journal 2015, 21, 13939-13942.

[34] Q. Zeng, L. Lin, J. Chen, Y. Lin, P. B. Barker, Z. Chen, J. Magn. Reson. 2017, 282, $27-31$.

[35] V. M. Rao Kakita, V. K. Shukla, M. Bopardikar, T. Bhattacharya, R. V. Hosur, RSC Advances 2016, 6, 100098-100102.

a) A. Bax, R. Freeman, J. Magn. Reson. 1981, 44, 542-561; b) M. Rance, G. Wagner, O. W. Sørensen, K. Wüthrich, R. R. Ernst, J. Magn. Reson. 1984, 59, 250-261.

[37] O. W. Soerensen, C. Griesinger, R. R. Ernst, J. Am. Chem. Soc. 1985, 107, 7778-7779.

[38] V. V. Krishnamurthy, Magn. Reson. Chem. 1997, 35, 9-12.

[39] L. Braunschweiler, R. R. Ernst, J. Magn. Reson. 1983, 53, 521-528.

[40] F. Zhang, R. Brüschweiler, J. Am. Chem. Soc. 2004, 126, 13180-13181.

[41] a) K. F. Morris, C. S. Johnson Jr, J. Am. Chem. Soc. 1992, 114, 3139-3141; b) C. S. Johnson Jr, Prog. Nucl. Magn. Reson. Spectrosc. 1999, 34, 203-256; c) G. A. Morris, in eMagRes, John Wiley \& Sons, Ltd, 2007; d) D. Sinnaeve, Concepts Magn. Reson. 2012, 40A, 39-65.

[42] E. O. Stejskal, J. E. Tanner, J. Chem. Phys. 1965, 42, 288-292.

[43] a) A. A. Colbourne, S. Meier, G. A. Morris, M. Nilsson, Chem. Commun. 2013, 49, 10510-10512; b) A. A. Colbourne, G. A. Morris, M. Nilsson, J. Am. Chem. Soc. 2011, 133, 7640-7643; c) M. Nilsson, M. A. Connell, A. L. Davis, G. A. Morris, Anal. Chem. 2006, 78, 3040-3045; d) M. Nilsson, G. A. Morris, Anal. Chem. 2008, 80, 3777-3782.

[44] J. E. Tanner, J. Chem. Phys. 1970, 52, 2523-2526.

[45] a) M. Nilsson, A. M. Gil, I. Delgadillo, G. A. Morris, Anal. Chem. 2004, 76, 5418-5422; b) M. Nilsson, A. M. Gil, I. Delgadillo, G. A. Morris, Chem. Commun. 2005, 1737-1739; c) N. Birlirakis, E. Guittet, J. Am. Chem. Soc. 1996, 118, 13083-13084; d) A. S. McLachlan, J. J. Richards, A. R. Bilia, G. A. Morris, Magn. Reson. Chem. 2009, 47, 1081-1085; e) B. Vitorge, D. Jeannerat, Anal. Chem. 2006, 78, 5601-5606. a) A. Botana, J. A. Aguilar, M. Nilsson, G. A. Morris, J. Magn. Reson. 2011, 208, 270-278; b) M. D. Pelta, G. A. Morris, M. J. Stchedroff, S. J. Hammond, Magn. Reson. Chem. 2002, 40, S147-S152.

[47] M. Nilsson, J. Magn. Reson. 2009, 200, 296-302.

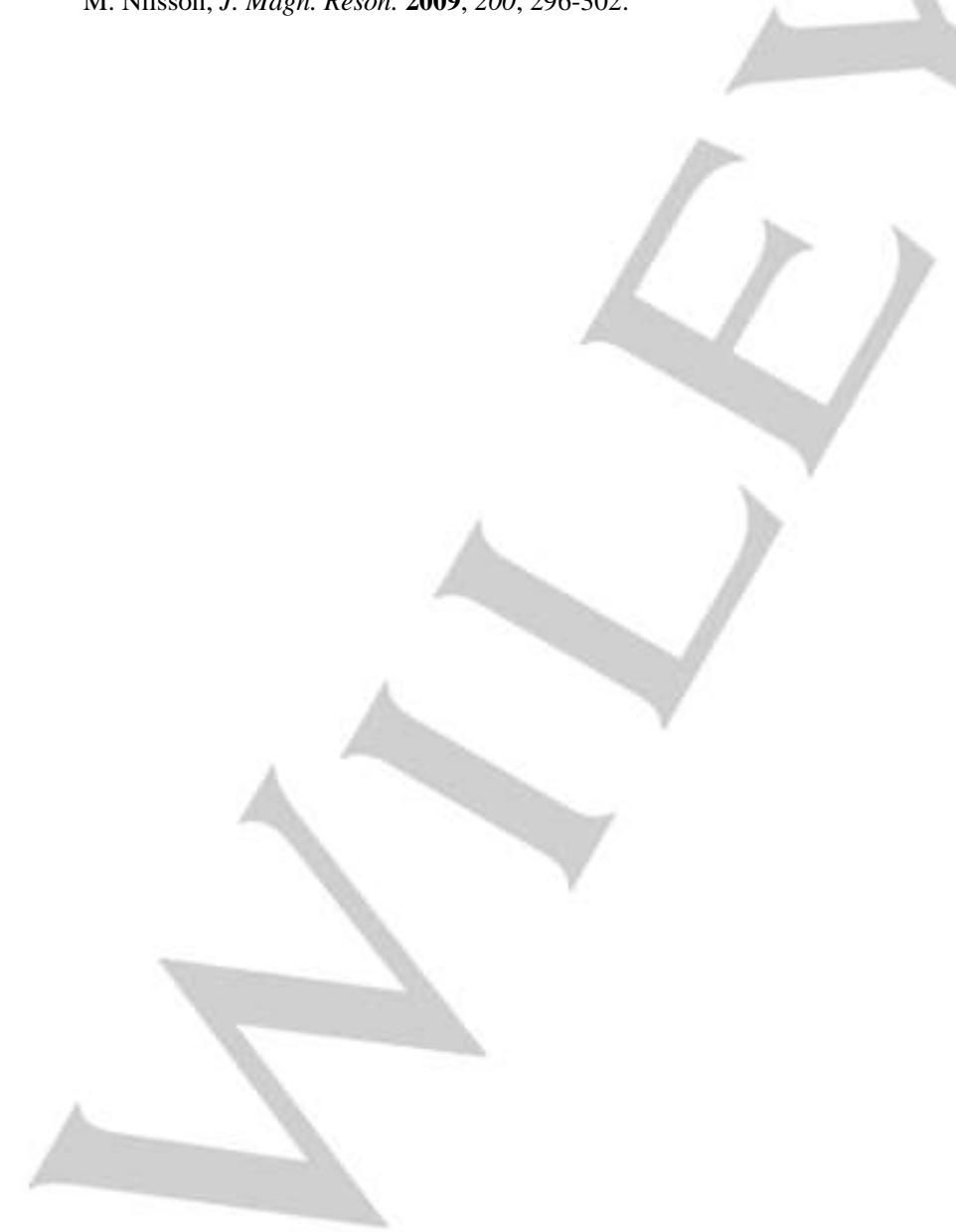

